\title{
Intermittent brain network reconfigurations and the resistance to social media influence
}

\author{
Italo'Ivo Lima Dias Pinto', Nuttida Rungratsameetaweemana ${ }^{2}$, Kristen Flaherty', \\ ${ }^{3}$, Aditi Periyannan ${ }^{1,4}$, Amir Meghdadi ${ }^{5}$, Christian Richard $^{5}$, Chris Berka ${ }^{5}$, Kanika \\ Bansal $^{1,6^{*}}$, \& Javier Omar Garcia ${ }^{1^{*}}$ \\ ${ }^{1}$ US DEVCOM Army Research Laboratory, Aberdeen Proving Ground, MD \\ ${ }^{2}$ The Salk Institute for Biological Studies, CA \\ ${ }^{3}$ Cornell Tech, New York, NY \\ ${ }^{4}$ Tufts University, Medford, MA \\ ${ }^{5}$ Advanced Brain Monitoring, Carlsbad, CA \\ ${ }^{6}$ Department of Biomedical Engineering, Columbia University, New York, NY
}

Keywords: social media, decision making, EEG, flexibility, network reconfigurations

${ }^{*}$ Co-senior and Corresponding authors: Kanika Bansal (phy.kanika@gmail.com) and Javier O. Garcia (javier.o.garcia.civ@army.mil)

\section{Abstract}

Since their development, social media has grown as a source of information and has a significant impact on opinion formation. Individuals interact with others and content via social media platforms in a variety of ways but it remains unclear how decision making and associated neural processes are impacted by the online sharing of informational content, from factual to fabricated. Here, we use EEG to estimate dynamic reconfigurations of brain networks and probe the neural changes underlying opinion change (or formation) within individuals interacting with a simulated social media platform. Our findings indicate that the individuals who show more malleable opinions are characterized by less frequent network reconfigurations while those with more rigid opinions tend to have more flexible brain networks with frequent reconfigurations. The nature of these frequent network configurations suggests a fundamentally different thought process between the individuals who are more easily influenced by social media and those who are not. We also show that these reconfigurations are distinct to the brain dynamics during an in-person discussion with strangers on the same content. Together, these findings suggest that network reconfigurations in the brain may not only be diagnostic to the informational context but also the underlie opinion formation.

\section{Author Summary}

Distinctive neural underpinnings of opinion formation and change during in-person and online social interactions are not well understood. Here, we analyze EEG recordings of the participants interacting with a simulated social media platform and during an in-person discussion using a network-based analysis approach. We show that the structure of network reconfigurations during these interactions is diagnostic of the opinion change and the context in which information was received. 


\section{Introduction}

Decision making is the internal process by which information is reduced to a categorical and actionable proposition (for review, see Gold \& Shadlen, 2007). In the brain, the decision making process has been described as a non-linear, context-dependent process that requires a variety of brain areas to receive and interpret information (e.g., sensory), establish value of this information, and then, based on prior experience and motivation, use a decision variable to produce the proposition and subsequently act (Fellows, 2004). One context that is currently and almost ubiquitously used as a source of information is social media, the suite of interactive online technologies that have become a mainstay of not only our everyday interactions but also current events and global happenings (Westerman et. al., 2014). Because of the ubiquitous nature of social media, the unbridled spread of information through it (Yoo, et. al. 2016), and the potentially negative consequences of it (Keles et. al., 2020), it is important to understand how it shapes our thoughts, influences our opinions, and impacts our future actions.

The neurological processes underlying the formation or changing of opinions due to social media exposure have been studied from the perspective of the presence and nature of biased content, and the way in which others interact with the information (e.g, likes, comments, retweets, etc.). Prior neuroscience work has specifically studied the effect of social influence on opinion formation and opinion change within the social media environment, where a network of brain regions including the striatum, orbitofrontal cortex, and temporoparietal junction appear to have a critical role in this decision making process (Cascio et al., 2015; Casado-Aranda et al., 2020; Sherman et al., 2016; Baek et al.,2021; Nakao et al., 2016; Falk et al., 2012; Falk \& Scholz, 2018; Kappes et al., 2020; Izuma \& Adolphs, 2013; Li et al., 2019; Klucharev et al., 2011). Specifically, the neural mechanism of opinion change due to social media use has been shown to integrate brain areas of the valuation, social pain/exclusion, and mentalizing systems which include the ventro-medial prefrontal cortex (VMPFC), striatum, medial prefrontal cortex (mPFC), dorsomedial prefrontal cortex (DMPFC), temporo-parietal junction (TPJ), posterior cingulate (PCC), medial tegmental gyrus (MTG), and anterior cingulate (ACC) (Cascio et al., 2015; Baek et al.,2021; Kappes et al., 2020; Falk et al., 2012). Other work has suggested that the popularity of content (Sherman et al., 2016) and the valence of the content plays a significant role in swaying opinion on these platforms (Baek et al., 2021). Due to the opportunity social media affords in rapidly disseminating information throughout the globe, it also creates an interesting glimpse into the complex human decision making process that impacts our everyday lives (Schmälzle et. al., 2017). Indeed, with the intensity and speed in which information spreads in this media convolved with the global scale, the contextual impact on decisions derived from platforms like these have had demonstrably profound impacts on society as a whole (Spinney, 2017).

Despite the understanding of the importance of these platforms in forming our decisions, it is still unclear how brain networks composed of regions, perhaps those associated with social media informational processing and influence, interact to produce opinion change. Importantly, it is also unclear how this process may be unique to brain processes underlying in-person interaction and free discussion. Network neuroscience provides a variety of tools to understand the complex network properties of the brain and has proven successful in describing a variety of behaviors 
(e.g., Bassett and Sporns, 2017 or Betzel and Bassett, 2017). For example, the rate at which networks within the brain rapidly reconfigure to support cognition has been found to be highly predictive of a variety of cognitive processes. Dynamic community detection, a technique used to distill complex connectivity patterns into time-varying labels of communities (i.e., clusters of nodes) has been successful in capturing variability in a variety of behaviors. This so-called flexibility has been used to describe the rate of motor learning (Bassett et al., 2011; Bassett et al., 2013; Gerraty et al., 2018; Li et al., 2019; Reddy et al., 2018), and associated with multi-tasking (Alavash et al., 2015; Shafiei et al., 2020; Thomas Yeo et al., 2015), pattern recognition (Telesford et al., 2016), language comprehension (Chai et al., 2016), thought rumination (Han et al., 2020; Lydon-Staley et al., 2019), adaptations to new stimuli or stress (Paban et al., 2019; Betzel et al., 2017), and working memory (Braun et al., 2015; Lauharatanahirun et al. 2020). Here, we have investigated the rapid fluctuations in network connectivity while individuals are exposed to an interactive social media platform containing factual and fake content and relate this to changes in opinions after exposure to this content, as we hypothesize that the flexible dynamics within the brain may be associated with complex decision making behind opinion change. Importantly, we provide a comparison to in-person discussion which allows us to disentangle the unique neural properties of this process. Our results provide preliminary evidence of unique neural features marking the cognitive processes supporting decision-making prompted by digital stimuli on a social media platform.

\section{Results}

We have investigated the neural correlates of complex decision making during online social media and in-person social interactions and assessed opinion change with questionnaires that asked participants for their opinions on several topical issues. Opinions on these topical issues were gathered before and after the interaction with a simulated social media platform and after in-person discussion of the content (Figure 1, see also Richard et. al., 2021). EEG was concurrently collected (see Supplemental Material Figure S1 for electrode montage) during the social media and in-person interactions and was analyzed to understand the rapid reconfigurations in EEG-derived brain connectivity matrices during the complex process of information gathering and opinion change and/or formation. Here, we used dynamic community detection, an algorithm that has previously been shown to successfully capture brain network reconfigurations associated with the variability in human behavior across a variety of tasks. We extended these findings by inspecting the temporal dynamics of node-pair community affiliations and comparing this metric between those that changed their opinions and those that did not across these social interaction conditions.

\section{Characterizing rigid and malleable opinionators in social media and in-person interactions}

Figure $1 \mathrm{~A}$ shows the experimental timeline, where, after arriving in the laboratory, subjects were presented with questionnaires that asked their opinions on particular real-world topical issues that included (i) travel based on social awareness and volunteerism, (ii) punishment after a murder trial, and (iii) decisions to vaccinate from disease before and after interaction with the social media platform as well as after the in-person discussion segment. EEG was recorded during these two interactive 
conditions, i.e., social media interaction and in-person discussion. Figure 1B-D shows the distribution of responses and the rate of opinion change across participants $(\mathrm{N}=132)$ following both conditions. First, with the social media platform, a majority of individuals did not change their opinion from the initial survey $(N=75)$; however, a total of 57 individuals changed their opinions, with the most individuals changing their opinion in the murder trial scenario $(\mathrm{N}=39)$. A small proportion of the individuals $(N=10)$ changed their opinions in two scenarios and were most likely to change their opinion on the travel and murder scenarios (Figure 1B). Figure 1C displays a more granular visualization of responses for each scenario, and similar to the design of the experiment which presented equally positive and negative coverage on an issue, there was a large diversity in opinion changes, validating the well-balanced affectual information within the platform. For example, even with the vaccination scenario, there were some individuals who changed their opinion toward not vaccinating after the social media interaction. Importantly, as well, is the fact that the change in prison time in the murder trial contributed to the largest changes, with 24 subjects changing the prison time after the social media interaction and 24 changing from guilty to not guilty.

We observed some similarities and differences in opinion change after in-person discussion. As shown in Figure 1D-E, the overwhelming majority of individuals $(73.5 \%, \mathrm{~N}=97)$ did not change their opinion, suggesting that the in-person discussion was less likely to affect one's opinion than social media interaction; however, the order of the questionnaires was the same across all individuals. This limitation does not allow us to disentangle the effect questionnaire order may have on our effects. Similar to the social media interaction condition, though, changes in opinion mostly occurred within the murder trial condition, accounting for $80 \%$ of the total changes in opinion after the discussion. Very few individuals changed their opinion in more than one scenario, accounting for only $8.6 \%$ of the total opinion change after the discussion.

Finally, to characterize the opinion change, overall, we estimated transition probabilities as presented in Figure 1F. These transition probabilities can give us a glimpse into the individuals that did and did not change their opinions following the social media interaction and in-person interactive conditions. As is shown in Figure $1 \mathrm{~F}, 56.8 \%$ of the subjects that did not change their responses after social media interaction, referred here as rigid opinionators, $74.7 \%$ of these rigid opinionators also kept their responses after in-person discussion. From the $43.2 \%$ of the subjects that changed their responses after the social media interaction, referred here as malleable opinionators, $28.1 \%$ changed their responses after in-person discussion as well. Importantly, of all the participants, $73.5 \%$ did not change their opinion after in-person discussion. Due to the distribution of those that did not change their opinion and those that did, we next compared the rigid and malleable opinionators within this population. 
A

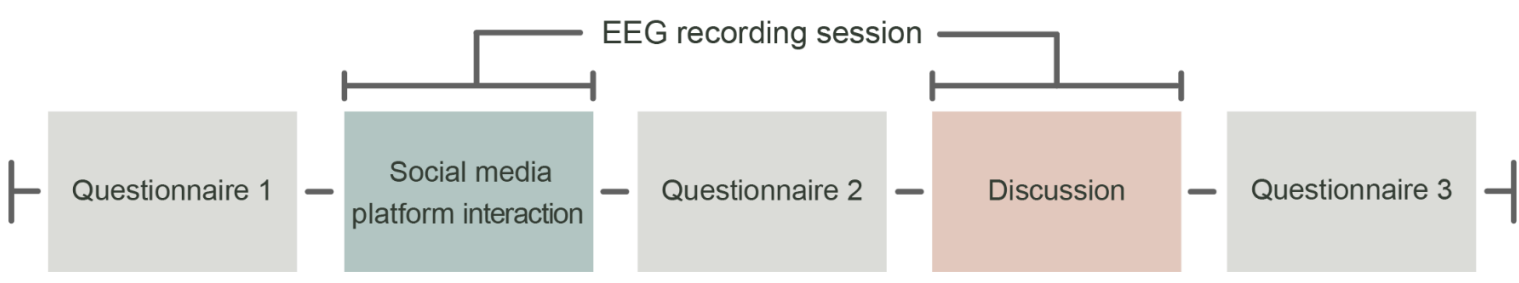

Social media platform opinion change

B

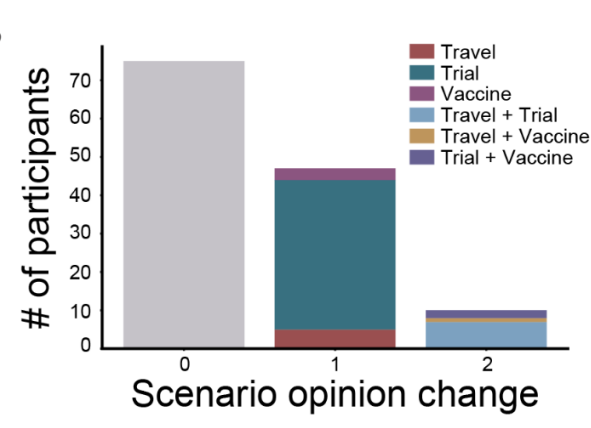

C Travel

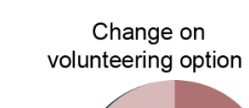

volunteering option

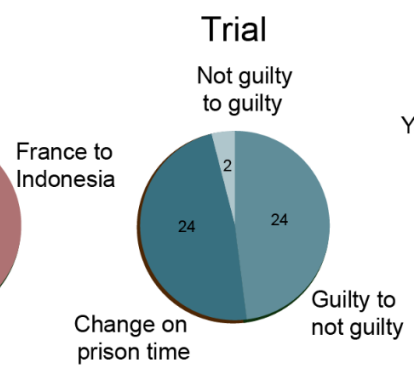

Indonesia to France

Discussion opinion change

D

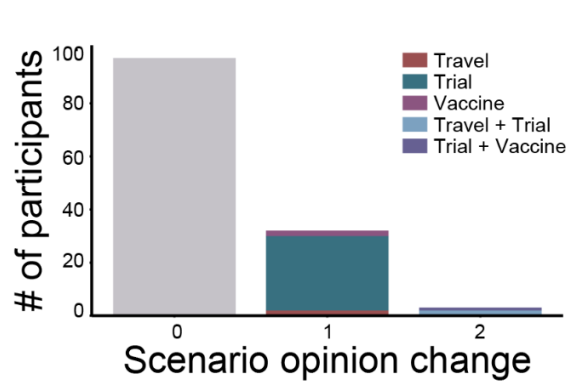

E Travel

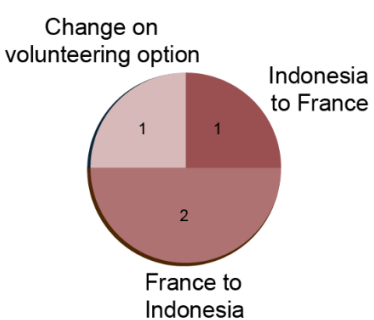

Trial

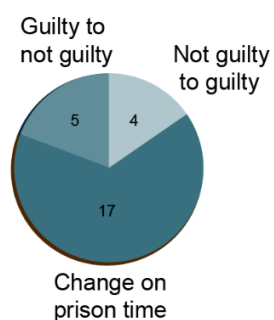

Vaccine

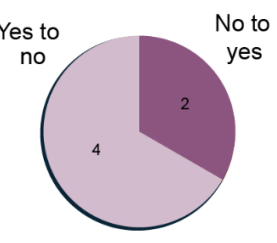

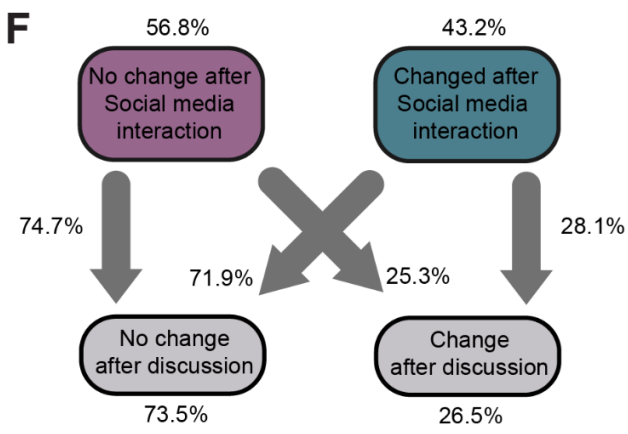

Figure 1: Experimental setup and opinion change quantification. (A) Timeline of the experimental design. The opinion changes of each subject were assessed through the application of a questionnaire before and after the subject interaction with the social media platform, in addition to in-person discussion. (B,D) Histogram of opinion change by scenario in social media interaction and in-person discussion, respectively. 0,1 , and 2 indicate the number of scenarios on which individuals change their opinion. Color legend indicates the scenario. (C,E) Pie charts indicating the direction of opinion change for all of the changes observed after the social media platform and in-person interactions. (F) Flow chart indicating the fraction of participants that changed their opinion from the social media platform interaction to in-person discussion. 


\section{Nodal Flexibility distinguishes rigid and malleable opinionators}

We hypothesized that complex decision making and information processing requires the reconfiguration of underlying brain networks. To test this hypothesis, we applied a dynamic community detection analysis to the EEG data and probed how network reconfigurations are associated with opinion change by directly comparing the rigid and malleable opinionators (Figure 2). This was accomplished in several steps. First, the dynamic community structure requires an estimate of the underlying statistical dependency between nodes. Here, we estimated this statistical dependence, or functional connectivity of the EEG using the pairwise weighted phase lag index (wPLI) separately for commonly studied EEG oscillations (i.e., delta (1-3 Hz), theta (3-7 Hz), alpha (8-13 Hz), beta (21-30), and gamma (25-40) bands) in non-overlapping $10 \mathrm{sec}$ time windows. This data-driven approach to functional connectivity exploits phase-based relationships within the data, yielding connectivity matrices that are reliable and less susceptible to some expected artifacts without requiring parameterization (Hardmeier et. al., 2014). Once calculated, the wPLI matrices were used to determine the community structure by modularity maximization using a Louvain algorithm (see Methods). This distilled the connectivity time evolving matrices into an average of 315 (SD = 102) time windows of community labels that represent the band-specific community affiliations of EEG sensors across time. 


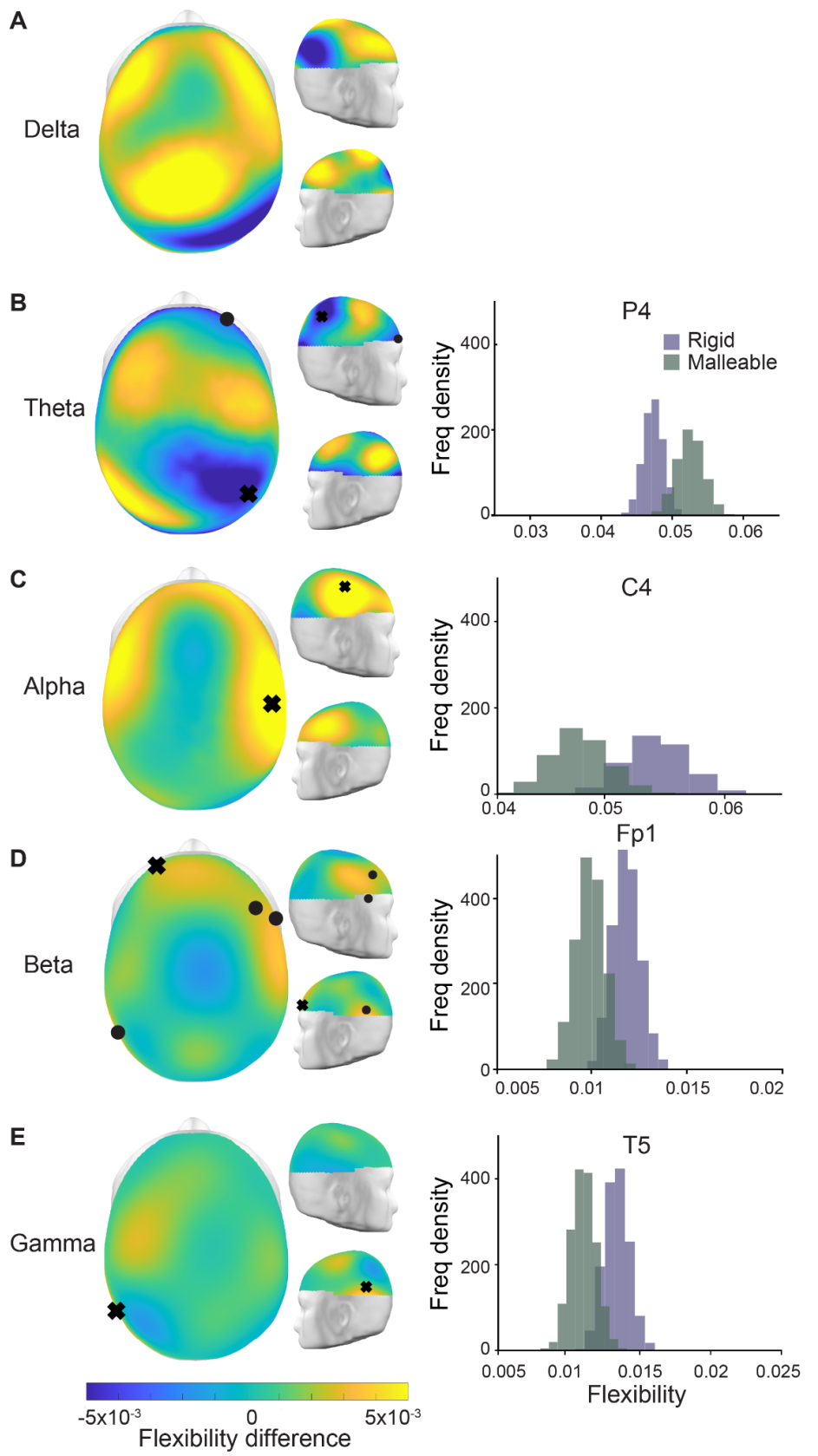

Figure 2: Flexibility differences between the rigid and malleable opinionator groups. (A-E) Topographic plots of each frequency band showing the difference of the mean flexibility between the two groups such that positive values (in yellow) indicate an increased flexibility for rigid opinionators. The black tokens (orbs, x's) indicate sensors with statistically significant differences in flexibility between the two groups as found via a bootstrap procedure (see Methods). On the right, we show representative bootstrap distributions of the mean flexibility of the sensors marked by an $\mathrm{x}$ for the two groups with rigid opinionators in purple and malleable opinionators group in green.

From these affiliations (i.e., distilled connectivity matrices), we estimated sensor (node) flexibility which is a measure of how much each node changes its affiliation across time in the opinion-rigid and -malleable individuals. Since the data is not balanced between the groups, we employed a bootstrap procedure to estimate the distributions of mean flexibility for each of the EEG sensors for both of the groups, 
and subsequently compared the node flexibility between rigid and malleable opinionators (see Methods). We observed that those rigid in their opinion showed significant increase in node flexibility in the alpha, beta, and gamma bands and decrease in flexibility in the theta band. Figure 2 shows the node flexibility differences, yellow (or blue) shades indicate an increased flexibility on the rigid (or malleable) group. An increased node flexibility in those with rigid opinions was observed in the higher frequency bands, with beta showing differences with statistical significance (bootstrap analysis, $p<0.05$ ) for sensors F8, F4, Fp1 and $\mathrm{T} 5$, gamma for $\mathrm{T} 5$, and alpha for $\mathrm{C} 4$. On the other hand, theta band presents a statistically significant decrease in node flexibility of the rigid opinionators on sensors P4 and Fp2. These results indicate that the dynamics of the synchronization-desynchronization processes, as measured by the wPLI coupled networks, play an important role in the underlying mechanism of opinion change during the social media interaction condition.

\section{Assessing dynamic changes in community structure}

We found that the node flexibility is informative as a neural marker of opinion change; however, it does not provide much information of the dynamic changes in the community structure to further understand the underlying network reconfigurations leading to opinion change. For example, one could ask how the links of the flexible nodes evolve with time and which other nodes couple and decouple with them more often during the task. In this regard, allegiance is a commonly used metric that captures the fraction of time two nodes share the same community affiliation, 0 for a pair of nodes that never share a community and 1 for nodes that are always in the same community. We estimated node allegiances for rigid and malleable opinionators and found that they do not differentiate the two groups (for further details, see Supplemental Materials and Figure S2). However, it is also unclear from allegiances alone whether more fine-grained temporal dynamics of network reconfigurations might differentiate these groups. To more finely understand the temporal evolution of node-pair affiliation change, we computed a new metric called intermittence.

Like allegiance, intermittence is a measure of the interaction between two nodes of the network; however, while allegiance captures the fraction of time two nodes belong to the same community, intermittence tracks how frequently the two nodes change their affiliation from the same to different and vice versa. In other words, intermittence differentiates two nodes' affiliation changes that occur in rapid bursts from affiliation changes that occur in longer-term after more static community affiliation similarity. Together, we may inspect allegiance as the likelihood for two nodes to be in the same community, and intermittence can inform us of the temporal nature of this relationship. 


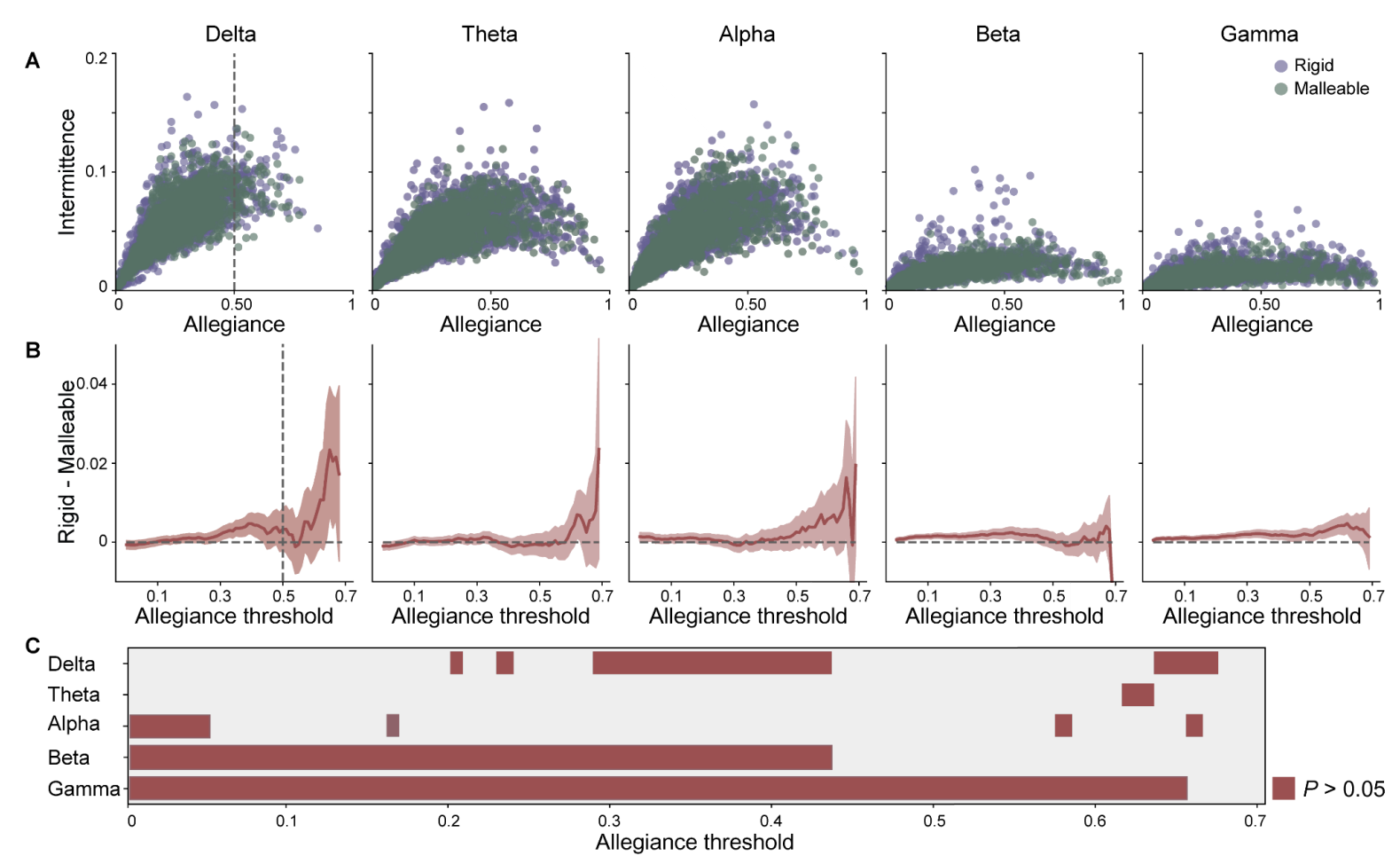

Figure 3: Comparing intermittence and allegiance in opinion change. (A) Scatter plots of the relationship between intermittence and allegiance for each frequency band of interest, where purple (or green) indicates channels of rigid (or malleable) opinionators. Dashed vertical line indicates the middle value of allegiance. (B) Bootstrapped difference (rigid - malleable) plots of intermittence of the subject groups for different threshold levels of allegiance for each frequency band of interest. Shaded region is 95\% confidence interval and the vertical dashed line indicates the midpoint of allegiance. (C) Allegiance thresholds that survive statistical comparison of rigid and malleable opinionators for each frequency band.

\section{Intermittence differentiates changes in opinion}

In exploring the intermittence metric, we first directly compare the allegiance and intermittence metrics for both rigid and malleable opinionators (Figure $3 \mathrm{~A}$ ). We observe for lower frequency bands (e.g., delta) that intermittence is more variable, spanning a wider range of values than higher frequency bands while the opposite is true for allegiance. Specifically for the delta band, values of allegiance larger than 0.6 are less frequent than observed for the other frequency bands and values of intermittence above 0.1 are more frequent than for the beta and gamma bands. On the other hand, inspecting higher frequency oscillations, we see intermittence is rarely above 0.05 but allegiance spans the entire range of possible values. This suggests that there is a higher propensity for more static network reconfigurations at higher frequencies than lower frequencies (e.g., compare Figure 3A gamma and delta). Importantly, with intermittence estimation, simply by its calculation, allegiance imposes an inherent restriction on its range of possible values. The maximum value of intermittence for a given pair of nodes is limited by the value of allegiance between those nodes, as the reader should understand that there cannot be more dynamic changes between nodes if they are rarely ever in the same community. Thus, given our observation that higher frequency bands (beta, gamma) had higher allegiance (that could allow for higher intermittence) in addition to the observed lower 
average intermittence, the finding that higher frequency bands show are even more striking, suggesting higher frequencies display very static network dynamics across groups.

In Figure 3B, we show the differences between the means in intermittence of the two groups. Critically, mean bootstrap distributions were calculated using only those points with associated allegiance values higher than the allegiance threshold indicated on the x-axis. The shaded area in Figure 3B represents a 95\% confidence interval and was obtained by a bootstrap procedure with 10,000 samples (see Methods). To summarize the statistical comparison between rigid and malleable opinionators at each of the frequency bands, Figure $3 \mathrm{C}$ shows those allegiance thresholds that display the statistical difference $(p<0.05)$.

Comparison of the mean intermittence between the two groups shows that the intermittence metric can delineate between rigid and malleable opinionators in each frequency band, but to a highly variable extent. For example, our results show that within the delta band, we observed statistically significant differences for the allegiance threshold range between 0.29 and 0.43 and a few other allegiance values accounting for more than $14 \%$ of the possible allegiance range values. For the theta band, there were minimal differences between groups observed accounting for only $2.8 \%$ of the total allegiance range. Within the alpha band, we observed, again, minimal differences between the groups accounting for less than $5 \%$ of allegiance thresholds; importantly, they were observed mostly at the lowest allegiance thresholds. The most robust differences between the rigid and malleable opinionators were observed within the beta and gamma bands. For the beta band, we observed significant differences between the groups in approximately $43 \%$ of the allegiance threshold range. The lowest $p$-values we observed between rigid opinionators group ( $\mathrm{M}=0.018$ a.u. $)$ and malleable opinionator ( $\mathrm{M}=0.017$ a.u.) was for the allegiance threshold of $0.12\left(p=2.7 \times 10^{-5}\right)$. The largest range of allegiance values with significant differences between the two groups were observed in the gamma band, accounting for nearly $65 \%$ of the entire allegiance range. The lowest $p$-value was observed at an allegiance threshold of 0.29 for rigid $(M=0.018$ a.u.) and malleable ( $\mathrm{M}=0.016$ a.u. $\left.p=3.6 \times 10^{-6}\right)$ individuals. Thus, it appears that intermittence successfully delineates rigid and malleable opinionators, but does so in a frequency band specific manner where the beta and gamma bands show the most robust differences as indicated by a wide range of allegiance thresholds for which the two groups have a significant difference in intermittence. In other words, intermittence can be used to characterize opinion change in band-specific oscillatory schemes, but it is still unclear whether this is a general opinion change phenomenon or if this may be specific to the context in which information is received (i.e., social media platform). Thus, we next explored how the malleable and rigid opinionators differ during in-person discussion. 


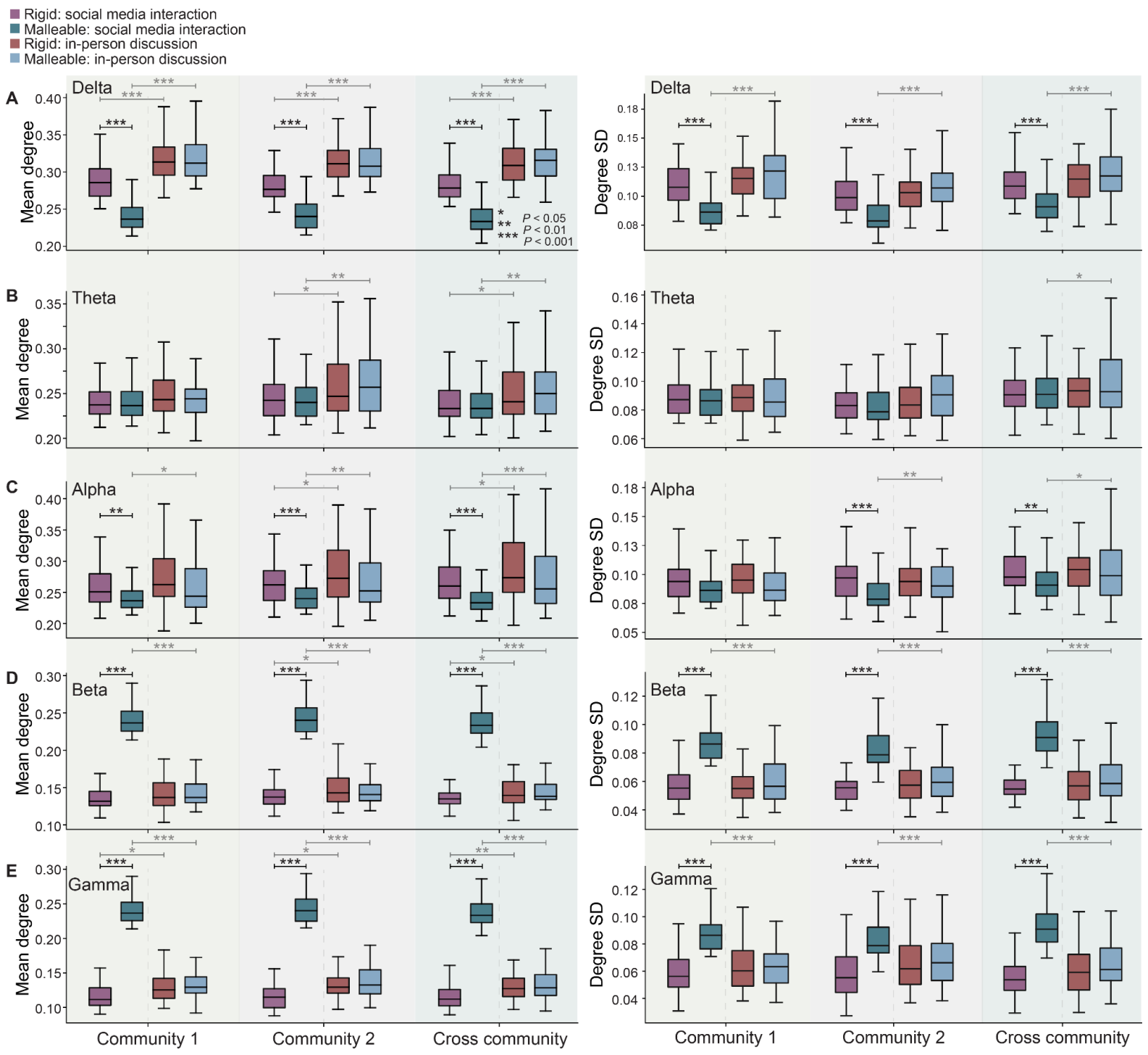

Figure 4: Social Media and face to face discussion comparison. (A-E) Mean (left column) and standard deviation (right column) of estimated degree within two consensus communities and across them for each frequency band (A) delta,(B) theta, (C) alpha, (D) beta, and (E) gamma. Each panel presents results for the rigid (purple-red) and malleable (blue-green) opinionators interacting with the Social Media platform and during in-person discussion. Statistical differences were performed with a Welch's t-test andare represented with an asterisk and are shown both within group and across social interaction contexts $\left({ }^{*} p<0.05,{ }^{* *} p<0.01\right.$, ${ }^{* * *}$ $p<0.001)$.

\section{Social media interaction and in-person discussion differences}

To determine the specificity of our findings to the social interaction context (e.g., social media vs in-person discussion), we sought to determine if network dynamics between malleable and rigid opinionators have similar structure during social media and in-person interactions. To carry out this comparison, we first derived a consensus community organization from the network reconfigurations (Doron et al., 2012) during social media interaction (removing the temporal aspect, see Supplemental Material) and used that as a reference to compare two different social interaction scenarios. We observed that on an average, during social media 
interaction, all the nodes can be divided into two large communities (Figure S3); one anterior region with 11 sensors (Fp1, F7, F8, T3, Fp2, F3, Fz, F4, C4, C3, Cz) and the other, a posterior region with 9 sensors (T4, T6, T5, O1, P3, Pz, P4, POz, O2).

We estimated how the connectivity of nodes within (and between) these communities change for the two groups (i.e., rigid and malleable) across two conditions of social interactions. For simplicity, we chose the nodal degree as a measure of connectivity of each node. The degree of a node is calculated as the sum of all its connections. We estimated the standard deviation and mean of nodal degrees within and between each community and compared their distributions for rigid and malleable opinionators for both the interaction conditions.

As shown in Figure 4, comparing the mean degree between rigid and malleable opinionators, we observed clear differences, during the social media interaction, but not as much during the in-person discussion. For example, the mean and standard deviation for malleable opinionators was significantly higher than those of rigid opinionators within the beta band $\left(p<1 \times 10^{-30}\right.$ for both communities and cross-community degree) and gamma band ( $p<1 \times 10^{-35}$ for both communities and cross-community) during social media interaction. Whereas, this difference was not present during the in-person discussion when comparing these groups (all $p>0.05)$.

Other observed differences in the social media condition were within the delta and alpha bands where those rigid in their opinions displayed significantly higher degree than those malleable in their opinions (delta: $p<1 \times 10^{-10}$ for both communities and cross-community, alpha: $p<1 \times 10^{-10}$ for both communities and cross-community) during social media interaction but not during in-person interaction. We also observed some differences between social interaction conditions (light gray horizontal bars, Figure 4), but were primarily for the malleable opinionators, especially in the alpha, beta and gamma bands. The delta band contained the most differences across social interaction context (social media interaction vs in-person discussion) while the beta and gamma bands contained the most differences in groups derived by opinion change. These results provide evidence to the uniqueness of neural dynamics during opinion change (or formation) in a social media interaction and suggest that social context and decision making trigger fundamentally different processes within the brain.

\section{Discussion}

Decision making is a complex internal process by which information is consumed and an action is executed, requiring the support of many interacting brain networks composed of a variety of functionally diverse regions within the brain (for review, see Fellows, 2004; Rilling and Sanfey, 2011; Wallace and Hofmann, 2021). The present study investigated the impact of informational context and its type on decision processes, specifically how social media and in-person discussion influences one's malleability to change one's mind on "highly shared" content in online platforms. Our findings have shown a large portion of individuals (e.g.,19.7\% in vaccination hesitancy) were susceptible to content displayed to them on a simulated social 
media platform while the same individuals were not susceptible to freeform and in-person discussions on the same topics. Using dynamic community detection (see Garcia et al., 2018 for a review) and estimating the temporal dynamics of network reconfigurations that occurred across several frequency bands, we found that the flexibility of specific sensors across the scalp could discriminate between those that are and are not influenced by content presented in a simulated social media platform. Importantly, rigid opinionators showed higher flexibility in sensors located within the frontal and posterior regions for the higher frequency bands (i.e., alpha, beta, gamma) whereas, the opposite effect is observed in theta band where higher flexibility was observed for the malleable opinionators within prefrontal and posterior sensors. Our results extended the flexibility findings to show that the vigor in which the network changes occurred was driving this effect with a new metric we call intermittence. Interestingly, intermittence within the higher frequency bands was more robustly diagnostic for different opinionators than lower frequency bands. Moreover, while our results did not show substantial change in opinion after in-person discussion (only 35 people changed their mind after in-person discussion), we also did not find any of the observed brain network reconfiguration changes during in-person interactiont condition.

\section{Network flexibility is a marker of opinion rigidity}

Our findings support the increasing evidence suggesting the importance of the rapid reconfigurations of brain networks in cognition, and specifically, here, decision making as it pertains to opinion change and/or formation (for review, see Shine and Poldrack, 2018). We used flexibility as the probability a particular node (i.e. sensor) changes its affiliation across time. Previous studies show that this type of network-defined flexibility in frontal brain regions is associated with faster motor learning (Basset et al., 2011), psychological resilience (Paban et al., 2019), chronic behavior change in addiction (Cooper et. al., 2019), enhanced working memory performance (Braun et al., 2015) -- which is also moderated by sleep (see Lauharatinahirun et al., 2020) -- and even improved adaptive problem solving skills (Barbey et al., 2018). Given the diversity in these behavioral findings and our extension to even social media influence, it is reasonable to attempt to understand how this metric may be highly sensitive to a variety of complex cognitive phenomena.

Indeed, the neuroscientific journey that has led to the importance of flexibility in neural behavior may be understood from several different perspectives, and it is currently thought to be the foundation to the human's unique ability to rapidly adapt to task demands. First, it should be noted that we have estimated network-based flexibility via a mathematically defined dynamic network approach (see Methods) and on its surface it should not be confused with concepts such as cognitive flexibility (Uddin, 2020) and neural flexibility (Yin \& Kaiser, 2021), but it can be complementary to both (Mattar et al., 2016). Cognitive flexibility refers to the executive functions that allow an individual to rapidly transition from task to task and has been found to be associated with improved performance in a variety of tasks and also is reduced in certain pathologies (Hanes et. al., 1995). In contrast, neural flexibility, while often used in relation to cognitive flexibility, refers to the brain's ability to rapidly shift across tasks and be recruited for a variety of activities (Uddin, 2020). Dynamics in the neural signal have previously been discarded as noise, but are now accepted as 
describing valuable variability in human behavior and even psychopathology (Uddin, 2020). Our findings not only add to this growing literature and support the network science approaches that can successfully capture this variability, but specifically, we have used dynamic community detection, an extension of the network science approach of functional modularity, that is a theoretically derived (but individualistic) technique to probe dynamic network changes via a distillation of dynamic connectivity matrices (Garcia et al., 2018). Our research adds to the general nature of this technique to capture the broadly cognitive, distributed and adaptive nature of the brain, the primary criteria for flexible brain regions and networks (Yin \& Kaiser 2021).

\section{Intermittent and persistent network reconfigurations are diagnostic of opinion rigidity}

Our results expand on the pervasiveness of the flexibility results. We show that, at least for complex, high level decision making, not only the rapidly evolving network reconfigurations (as measured by flexibility) are important but also the fast dynamics of intermittent linking (same nodes linking in an on-off fashion) between modules are more diagnostic of social media influence on one's opinion. Dynamic community detection has proved to be an effective tool to explore temporal patterns in systems represented by complex networks and a key aspect for this framework is the determination of the temporal resolution of the dynamic communities (Telesford et. al. 2016). A systematic way to determine a temporal resolution which leads to behaviorally relevant network description of the brain can be achieved by modularity maximization (Garcia et. al. 2018). Based on the dynamic communities obtained through the modularity maximization algorithm, we explore the temporal patterns of network links and how community allegiances of the network nodes change across time.

A critical feature of our findings is the fact that the temporal profiles of the estimated community structure is more diagnostic (e.g., intermittence) than simply the fact that dynamic network reconfigurations occur. The temporal profile of interactions has a fundamental importance on a wide range of phenomena such as the dynamics of neuron populations that lead to seizures (Jirsa et. al. 2014, Rungratsameetaweemana et al, 2021), weather models and turbulent systems such as the Lorenz attractor (Ruelle 1976) and the many synchronization phenomena in which many units share the same temporal profile (Pikovsky et. al. 2001; Strogatz, 2000). From the point of view of dynamical systems, processes of opinion spreading have been extensively studied using models such as the Voter (Holley and Liggett, 1975) and Majority rule models (Krapivsky \& Redner, 2003), suggesting a complex interactive scheme that gives rise to opinion formation and change. Interestingly, recent findings suggest that information sharing and spreading occurs at a faster pace in social media platforms than in-person social contacts and explores the effects of these two time scales in a consensus formation model (Ding et. al., 2018). With our approach, we explore this opinion change phenomenon at an individual level using the complex networks framework to identify connectivity patterns of EEG data that are diagnostic to an opinion change process during a social media interaction. In aggregate, these findings coupled with our current results, suggest similar operations at both the neural level and population level. Recent findings suggest the brain (as well as other complex systems) operate outside of the 
boundaries of a particular spatial scale (Cocchi et. al., 2017). Perhaps, our results are the consequence of information spread, whether within a single brain or across human interactions and are suggestive of a scale-free phenomenon (Mahmoodi et. al., 2017). Indeed, there are many complex systems that express this scale-free behavior; however, it should be noted that recent findings have even shown that this universal principle is flexible (Bansal et. al. 2021).

\section{Network reconfigurations and oscillatory specificity suggest a complex operation within the brain in opinion change}

Despite the increasing efforts to understand the neural processes that underlie deliberation and decision making, much on this subject remains unclear; however, important findings from the literature in EEG oscillations, evidence accumulation, valuation and identity may play critical roles in understanding our results. First, we have shown that intermittence effects are more robust at higher frequencies than lower frequencies in the observable EEG oscillatory scheme (e.g., gamma vs theta). We also show that the delta band is most diagnostic for social interaction context. Oscillations emanating from the brain, as measured with EEG, are a consequence of short- and long-range connections within the brain that interact to give rise to cognitive capabilities (Buzsáki 2006). Importantly, the slower oscillations mostly represent the coordination of distal regions within cortex and sometimes even modulate higher frequency oscillations within the brain (e.g., Bragin et al., 1995; Chrobak and Buzsaki, 1998; Leopold et al., 2003; Schroeder and Lakatos, 2009; Canolty et al., 2006; Buzsáki and Wang, 2012; more recent ones). In other words, oscillatory activity and the associated cognitive functions rely on the global coordination of local processes (Cavanaugh \& Frank, 2014; Rungratsameetaweemana et al., 2018). Within this context, it would suggest that our results could be a consequence of both, where the most critical "intermittence" effects were observed within the delta and gamma bands, "flexibility" effects were most critical in the beta band, and social interaction context was most observed within the delta band.

This broad coordination of neural communication in the brain gives rise to specific cognitive functions, and our results could reflect several different operations at play. First, our findings show the most significant results in flexibility within the beta band. The beta band is often associated with motor behavior (Khanna \& Carmena, 2015), but has also been proposed to carry a more prominent role in maintaining motor or cognitive states (Engel \& Fries, 2010); interestingly, beta band dynamics have even been associated with the accumulation of evidence in the sensorimotor network in a vibrotactile decision task (Haegens et. al. 2011). Moreover, fMRI and transcranial magnetic stimulation (TMS) studies have extensively implicated the so-called value system in decision making, a system that is engaged when weighing the potential benefits of a particular decision route. Critical components of this system are thought to include the ventromedial prefrontal cortex (VMPFC) and ventral striatum (VS). These regions, within social contexts, have been linked to susceptibility to social influence from peers (for a comprehensive review see Falk \& Scholz, 2018). More broadly in EEG, several frequency bands have been implicated in decision making (e.g., Nakao et. al. 2019), but often show some specificity in frontal and parietal regions (e.g., Golnar-Nik et. al. 2019). Interestingly, a recent study inspecting long 
range temporal correlations (LRTC) in EEG recordings has shown a relationship between theta to alpha bands and the abstract concept of self identity and identity confusion (Sugimura et. al. 2021). Due to the highly complex decision, speculation, and potential action, our results most likely indicate a complex coordination and reconfiguration of networks within the brain, across several frequency bands and reflect coordination of these processes including evidence accumulation, valuation and even internal reflection on identity. Future research is needed to disentangle these processes and influences on decision making context, especially within the social media and in-person social contexts studied here.

\section{Methodological limitations}

Often with ecologically valid research, there is a limitation in understanding all elements that have contributed to our findings. For example, each subject was able to navigate freely through the social media content, and there was no control on how much time was spent on each content. Our results don't take into account any content-specific processes or differences on individual social media interaction, nor do they consider the controversial and emotional aspects of the different scenarios presented for the subjects. Within this context, our results are merely the first step toward understanding the dynamic reconfigurations within the brain and how different context and content interact to give rise to opinion change, highlighting the difference between in-person discussion and social media interaction. Future studies are needed to understand full contributions to session and subject level variability as well as disentangle the "intermittence" results as potentially marking different internal processes while interacting with social media or different strategies deployed for social media interaction.

\section{Conclusion}

The current study used a complex network based framework (dynamic community detection) to investigate the relationship of brain dynamics during social media interaction with the opinion change and/or formation processes. Our results indicate that the rapidly evolving network dynamics in delta, beta and gamma bands are the markers of influence of social media platform interaction on opinions in a range of scenarios, such that the slower dynamics is associated with individuals who are more likely to change their opinion, i.e, malleable opinionators. We also introduced a new metric called intermittence to assess differences in the observed faster or slower network dynamics. Estimating the intermittent and persistent network changes (as measured via intermittence), our results suggest that the functional brain network structure for malleable opinionators also show differences when interacting with social media platforms compared to in-person discussions. Together, our results suggest unique decision making operations during social media interaction and represent trait-like dynamics in individuals that are rigid or malleable in their decisions.

\section{Methods}

\section{Participants}

The data was collected from a cohort of 123 healthy participants between the ages of 18-40 years. Subjects were screened and the ones diagnosed with sleep, 
psychiatric, neurological, eating, behavioral (including Attention Deficit Disorder), or cardio-pulmonary/vascular disorders, uncontrolled blood pressure, heart disease, HIV+/AIDS, head trauma within the past 5 years, regular use of prescription drugs that can alter EEG or impair their ability to participate, use of illegal drugs (recreational and medical marijuana users were not excluded), excessive use of nicotine, alcohol and/or caffeine, untreated vision or hearing issues, pregnant or nursing, and inadequate familiarity with the English language were not included. For more information on the recruiting procedure we refer the reader to (Richard et. al., 2021). The data acquisition for this study using human participants was reviewed and approved by Alpha IRB and Air Force Research Laboratory (AFRL). The participants provided their written informed consent to participate in this study.

\section{Procedures}

For the experimental timeline and descriptive analysis of behavioral results (e.g., opinion change), see Figure 1. Briefly, after arriving in the laboratory, subjects were presented with questionnaires that asked their opinions on particular real-world scenarios found commonly on social media platforms. Questionnaires were presented before and after interaction with the simulated social media platform as well as after the in-person discussion segment. EEG was recorded during these two interactive conditions, i.e., social media interaction and in-person discussion.

\section{Scenarios}

Three hypothetical scenarios were presented to the subjects. During each session, subjects were exposed to the contents of three scenarios. The opinions of each subject in the scenarios were accessed through questionnaires that were delivered before and after interaction with a social media simulation software. The following details the three scenarios analyzed in this study.

Free travel destinations. Within this scenario, subjects could choose between two locations (Paris, France, or Sulawesi Island, Indonesia) for an all-paid one-week vacation where each location was vulnerable to different dangers; Paris could have large protests and sporadic violence and Sulawesi Island had the potential for a destructive tsunami. Articles presented to the subjects mentioned these dangers and mentioned how nice the locations were to visit, with equal representation. The subjects were also given the opportunity to volunteer, in support of the rebuilding effort following the catastrophic damage; they were also asked how much time they were willing to dedicate to the rebuilding effort.

Murder trial. In this scenario, subjects were asked to imagine themselves in the jury of a trial, which was based on a true story. After receiving information about a case in which a young female college student was murdered, they were asked whether the young man accused of murder should be considered guilty or not, and if guilty, the length of the sentence or death.

Vaccinations. In this scenario the subjects were asked about vaccinating one of two hypothetical children after the older one started to show development impairments after being vaccinated. The questionnaire presented only the binary question if the subject would vaccinate or not their second child, and the online content was equally for or against vaccinations. 


\section{Behavioral metrics}

The subjects answered questions about each of the hypothetical scenarios and oopinion changes on the topics of the three scenarios were evaluated to determine the likelihood of opinion change. . Of the three scenarios, the vaccination scenario was the only completely binary response as the travel and murder scenarios included followup questions that were not binary. Thus, several steps were completed to include all three scenarios in the analysis and construct a behavioral metric of opinion change.

For the three scenarios analyzed in this study, the social media opinion change was coded as either a 0 (no change) or a 1 (change), indicating a change in opinion relative to the previously answered questionnaire. In other words, for the social media interaction, change was measured relative to the first questionnaire and for the in-person discussion condition, opinion change was assessed relative to the second questionnaire (completed after social media interaction). Change was coded as follows: for the travel scenario, a response was considered a change (1) in opinion if any of the following were true: (i) the subject changed the destination choice from France to Indonesia or vice versa, (ii) the subject modified their decision to volunteer, or (iii) the subject changed the amount of time they would volunteer. For the murder scenario, a response was considered a change if any of the following were true, (i) the subject changed their opinion from guilty to not guilty and vice versa, (ii) the subject changed their opinion on prison time or punishment. For the vaccination scenario, the responses were either yes or no, so a response was considered a change if it did not match the previous response. If the answers are all identical to the previous survey, then the response was coded as a 0 , or no change. The social media opinion change score for each subject was estimated as the sum of the social media opinion change scores of each individual scenario and could be $0,1,2$, or 3 , where a 3 represents a change in every scenario and 0 in none.

\section{EEG Analysis}

\section{Preprocessing}

EEG was acquired using the B-Alert R X24 wireless sensor headset (Advanced Brain Monitoring, Inc., Carlsbad, CA, United States) placed on the subjects before the subjects interacted with the social media platform. The headset is composed of 20 EEG sensors located according to the International 10-20 montage at Fz, Fp1, Fp2, F3, F4, F7, F8, Cz, C3, C4, T3, T4, T5, T6, O1, O2, Pz, POz, P3, and P4. Reference sensors were linked and located behind each ear along the mastoid bone region. The sampling rate was $256 \mathrm{~Hz}$ and the signal was filtered through a high band pass filter at $0.1 \mathrm{~Hz}$ and a low band bass, fifth order filter, at $100 \mathrm{~Hz}$. To insure high quality data was collected, a maximum allowable impedance was set to $40 \mathrm{k}$. Next, the data was band-pass filtered within common frequency bands including delta $(1-3 \mathrm{~Hz})$, theta $(3-7 \mathrm{~Hz})$, alpha $(8-13 \mathrm{~Hz})$, beta (21-30) and gamma (25-40) using a low order (3) zero-phase forward and reverse digital IIR filter in Matlab (Mathworks, Inc.). 


\section{Functional Connectivity Analysis}

To estimate the functional connectivity of the EEG recordings we calculated the pairwise weighted Phase Lag Index (wPLI) within each frequency band of interest, which is known to be highly sensitive to linear and nonlinear interactions (Imperatori et al. 2019). For each sensor, the EEG (already band-wise filtered) was partitioned in $L$ windows with duration 10s. Each time window was used to calculate a matrix in which each entry $A_{i j}$ accounts for the weighted Phase Lag Index (wPLI) (Vinck et al., 2011) for the pair of sensors $i$ and $j$, calculated as:

$$
\Phi \equiv \frac{|E\{\Im\{X\}\}|}{E\{|\Im\{X\}|\}}
$$

where $E\{$.$\} denotes the expected value and \mathfrak{I}\{X\}$ is the imaginary part of the cross-spectrum of the EEG recordings of sensors $i$ and $j$. The temporal layers obtained by the described procedure were then used for the dynamic community detection analysis described in the next section. Importantly, though, the number of windows $(L)$ were variable across subjects, with a mean $L$ across subjects of 372 $(\mathrm{SD}=100)$.

\section{Community Detection and Network Dynamics Metrics}

While human brain mapping efforts have demonstrated a relationship between spatial specificity and cognitive functions, techniques rooted in network science provide a useful framework for characterizing and understanding the spatiotemporal dynamics of the functional systems subserving cognition (Bassett \& Sporns, 2017). One of the core concepts at the basis of network science is network modularity, which is the idea that neural units are structurally or functionally connected forming modules or clusters (Garcia et al., 2018). This organization allows for the system to perform both local-level exchanges of information, while maintaining system-level performance. Here, we examine whether a particular node's propensity to change communities (i.e., flexibility) was related to change in opinions after interaction with a social network platform. To measure such changes in network communities during the interaction with the social media platform, a multilayer community detection analysis was employed (Bassett et al., 2011; Mucha et al., 2010) on the aforementioned wPLI estimates. This method uses a Louvain algorithm to maximize modularity (Blondel et al., 2008) to define functional communities and is completed in several steps. First, it relies on two parameters, $\gamma$ and $\omega$, so called structural and temporal parameters of the analysis. We swept the parameter space from .5-4 for each parameter, subject, and segment and compared the mean estimated modularity value $Q$ to a shuffled null dataset. We chose a parameter set that on average produced more than 1 community and was the highest difference in modularity from the estimated modularity from the shuffled null dataset (see Garcia et al., 2020; Garcia et al., 2020 for a similar procedure). Due to the non-deterministic nature of the analysis, the chosen optimization procedure was repeated 100 times, since the algorithm is susceptible to multiple solutions (Good et al., 2010). From these multiple iterations, the following community metrics were computed: (i) flexibility, or proportion of time during which each node switches to a different community assignment; (ii) allegiance, related to how long two nodes are connected to each other during the task, and a new proposed metric (iii) intermittence, defined as how rapidly two nodes connect and disconnect through communities. Those 
metrics were calculated for each of the 100 iterations, and our results used the mean value for all the iterations. In more concrete terms, the flexibility of each node corresponds to the number of instances in which a node changes community affiliation, $g$, normalized by the total possible number of changes that could occur across the layers $L$. In other words, the flexibility of a single node $i, \xi_{i}$, may be estimated by

$$
\xi_{i}=\frac{g_{i}}{L-1} .
$$

Allegiance is a metric calculated for each pair of nodes and accounts for the proportion of the total time a pair of nodes belongs to the same community, and is defined as:

$$
\text { Allegiance }_{j k}=\frac{1}{L} \sum_{t=1}^{L} \delta_{C_{j}(t) C_{k}(t)},
$$

where $\delta$ denotes the Kronecker delta and $C_{l}(t)$ denotes the community which contains the node $l$ at time $t$. Therefore, $\delta_{C_{j}(t) C_{k}(t)}$ equals 1 if the nodes $j$ and $k$ are in the same community at time layer $t$ and equals 0 otherwise.

Further, to account for the temporal dynamics of allegiance, we proposed a new metric, intermittence, which tracks how frequently the two nodes change their affiliation from the same to different and vice versa. Intermittence is defined as:

Intermittence $_{j k}=\frac{1}{L-1} \sum_{t=1}^{L-1} \delta_{C_{j}(t) C_{k}(t)}\left[1-\delta_{C_{j}(t+1) C_{k}(t+1)}\right]+\delta_{C_{j}(t+1) C_{k}(t+1)}\left[1-\delta_{C_{j}(t) C_{k}(t)}\right]$. 


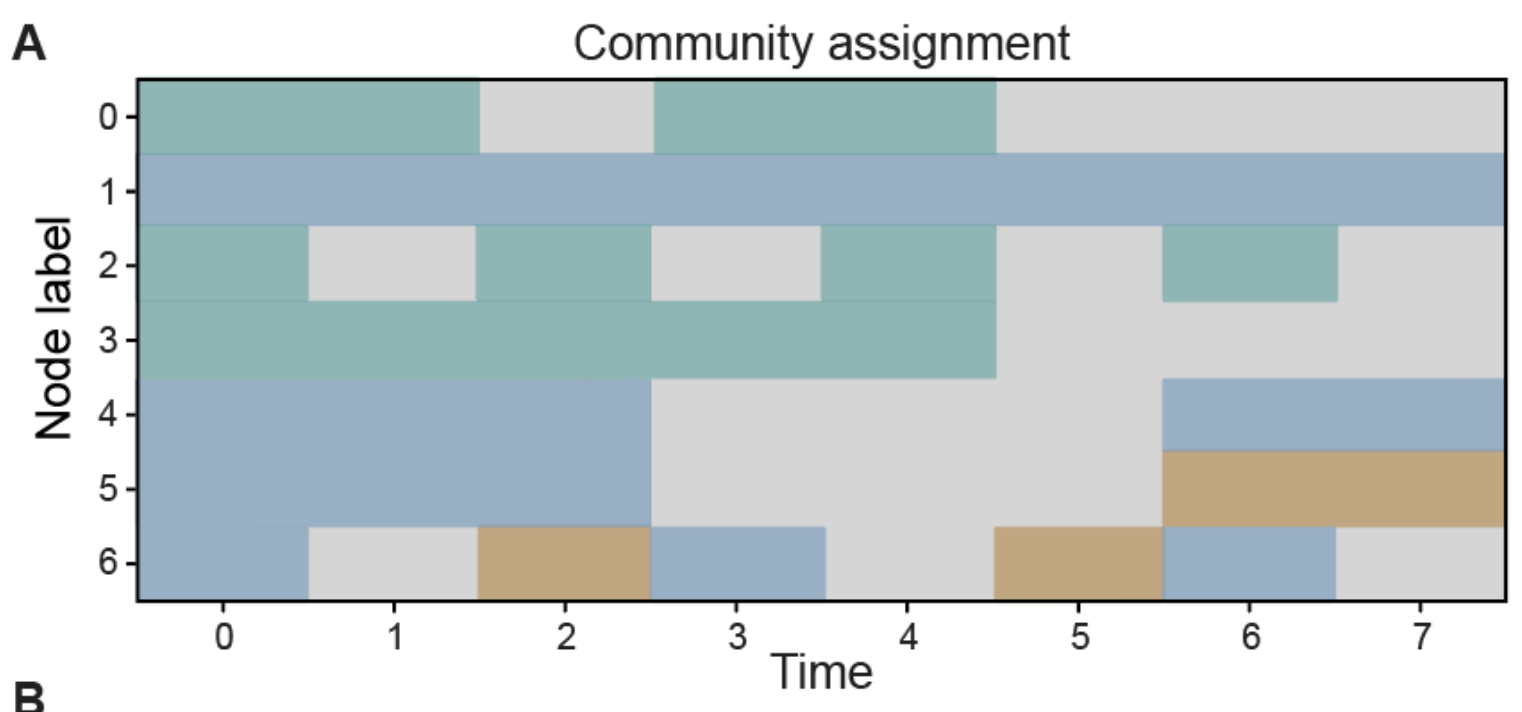

B

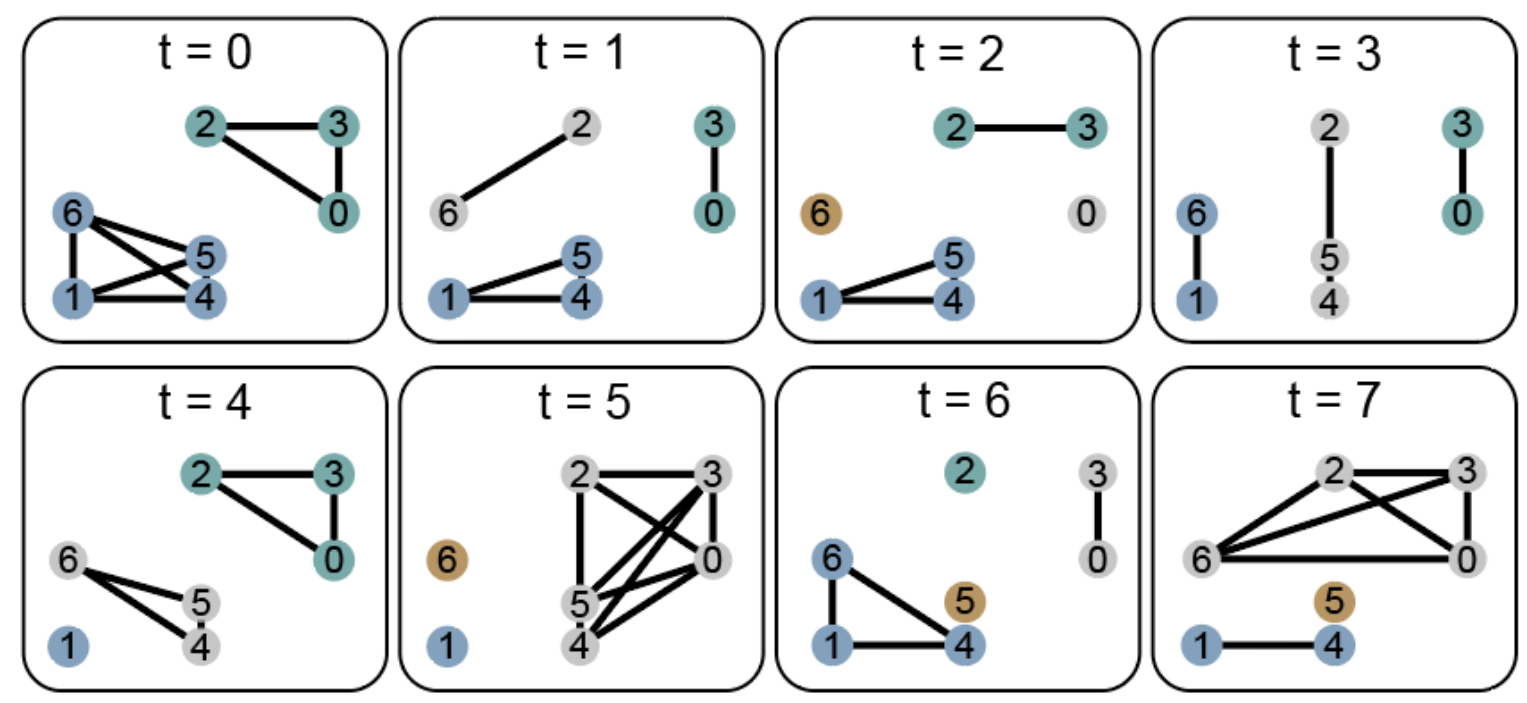

Figure 5: Flexibility, Allegiance, and Intermittence. (A) Example of a community structure assignment for 8 time layers. (B) Representation for each time layer of the community structure on $(A)$.

To visualize the concept of intermittence consider the example in Figure 5. First observe that the allegiance between nodes 1 and 4 is equal to the allegiance of nodes 2 and 3 , however the link between nodes 1 and 4 is present for two large continuous epochs while the link between nodes 2 and 3 is connected for many short epochs, this characterizes the intermittence between nodes 2 and 3 as larger than the intermittence between nodes 1 and 4 . Consider now the nodes 5 and 6 , both have the same allegiance with node 1 , however since node 6 changes its community assignment more often, its flexibility is higher than the flexibility of node 5 . Observe that unlike intermittence, flexibility is a property of the node and is not calculated for individual links of the nodes. 


\section{Statistical comparisons}

Two types of statistical comparisons were completed within the manuscript. Primary comparisons were between two groups, of unequal sizes, so-called rigid and malleable opinionators. Due to the unequal sizes, bootstrap distributions (Wehrens et. al., 2000) were estimated and used to estimate p-value and $95 \%$ confidence intervals (Figures 2 and 3). For this method, 10,000 drawings (with replacement) were made within each group for each node (Figure 2) or across nodes (Figure 3 ) and distributions of the value (e.g., flexibility) or distributions of differences (e.g., intermittence - allegiance) were estimated. This process generated different distributions, for which 95\% confidence intervals were then estimated. In the final analysis, Welch's t-test was used to compare the two group means, as it is robust to potential variance differences between groups.

\section{Acknowledgements}

This research was sponsored by the US DEVCOM Army Research Laboratory and was completed under Cooperative Agreement Numbers W911NF2020067 (I.L.D.P.), and W911NF-17-2-0158 (K.B.). The views and conclusions contained in this document are those of the authors and should not be interpreted as representing the official policies, either expressed or implied, of the US DEVCOM Army Research Laboratory or the U.S. Government. The U.S. Government is authorized to reproduce and distribute reprints for Government purposes notwithstanding any copyright notation herein.

\section{References}

Gold, J. I., \& Shadlen, M. N. (2007). The Neural Basis of Decision Making. Annual Review of Neuroscience, 30(1), 535-574. https://doi.org/10.1146/annurev.neuro.29.051605.113038

Fellows, L. K. (2004). The cognitive neuroscience of human decision making: a review and conceptual framework. Behavioral and Cognitive Neuroscience Reviews, 3(3), 159-172. https://doi.org/10.1177/1534582304273251

Westerman, D., Spence, P. R., \& Van Der Heide, B. (2014). Social Media as Information Source: Recency of Updates and Credibility of Information. Journal of Computer-Mediated Communication, 19(2), 171-183. https://doi.org/10.1111/jcc4.12041

Keles, B., McCrae, N., \& Grealish, A. (2020). A systematic review: the influence of social media on depression, anxiety and psychological distress in adolescents. International Journal of Adolescence and Youth, 25(1), 79-93. https://doi.org/10.1080/02673843.2019.1590851

Yoo, E., Rand, W., Eftekhar, M., \& Rabinovich, E. (2016). Evaluating information diffusion speed and its determinants in social media networks during humanitarian 
crises. Journal of Operations Management, 45, 123-133. https://doi.org/10.1016/i.jom.2016.05.007

Spinney, L. (2017). The shared past that wasn't: How Facebook, fake news and friends are altering memories and changing history. Nature, 543(9), 168-170. https://www.doi.org/10.1038/543168a.

Schmälzle, R., O'Donnell, M. B., Garcia, J. O., Cascio, C. N., Bayer, J., Bassett, D. S., Vettel, J. M., \& Falk, E. B. (2017). Brain connectivity dynamics during social interaction reflect social network structure. Proceedings of the National Academy of Sciences of the United States of America, 114(20), 5153-5158. https://doi.org/10.1073/pnas.1616130114

Falk, E., \& Scholz, C. (2018). Persuasion, Influence, and Value: Perspectives from Communication and Social Neuroscience. Annual Review of Psychology, 69(1), 329-356. https://doi.org/10.1146/annurev-psych-122216-011821.

Fleming, S. M., Van Der Putten, E. J., \& Daw, N. D. (2018). Neural mediators of changes of mind about perceptual decisions. Nature Neuroscience, 21(4), 617-624. https://doi.org/10.1038/s41593-018-0104-6.

Kaplan, J. T., Gimbel, S. I., \& Harris, S. (2016). Neural correlates of maintaining one's political beliefs in the face of counterevidence. Scientific Reports, 6(December), 1-11. https://doi.org/10.1038/srep39589

Edelson, M. G., Dudai, Y., Dolan, R. J., \& Sharot, T. (2014). Brain substrates of recovery from misleading influence. Journal of Neuroscience, 34(23), 7744-7753. https://doi.org/10.1523/JNEUROSCl.4720-13.2014

Izuma, K., Matsumoto, M., Murayama, K., Samejima, K., Sadato, N., \& Matsumoto, K. (2010). Neural correlates of cognitive dissonance and choice-induced preference change. Proceedings of the National Academy of Sciences of the United States of America, 107(51), 22014-22019. https://doi.org/10.1073/pnas.1011879108

Colosio, M., Shestakova, A., Nikulin, V. V., Blagovechtchenski, E., \& Klucharev, V. (2017). Neural mechanisms of cognitive dissonance (Revised): An EEG study. Journal of Neuroscience, 37(20), 5074-5083. https://doi.org/10.1523/JNEUROSCI.3209-16.2017

Doré, B. P., Cooper, N., Scholz, C., O'Donnell, M. B., \& Falk, E. B. (2019). Cognitive regulation of ventromedial prefrontal activity evokes lasting change in the perceived self-relevance of persuasive messaging. Human Brain Mapping, 40(9), 2571-2580. https://doi.org/10.1002/hbm.24545

Bai, Y., Nakao, T., Xu, J., Qin, P., Chaves, P., Heinzel, A., Duncan, N., Lane, T., Yen, N. S., Tsai, S. Y., \& Northoff, G. (2016). Resting state glutamate predicts elevated pre-stimulus alpha during self-relatedness: A combined EEG-MRS study on "rest-self overlap." Social Neuroscience, 11(3), 249-263. https://doi.org/10.1080/17470919.2015.1072582 
Wokke, M. E., Cleeremans, A., \& Ridderinkhof, K. R. (2017). Sure i'm sure: Prefrontal oscillations support metacognitive monitoring of decision making. Journal of Neuroscience, 37(4), 781-789. https://doi.org/10.1523/JNEUROSCI.1612-16.2016

Casado-Aranda, L. A., Venkatraman, V., Sánchez-Fernández, J., \& Luque-Martínez, T. (2020). Does Partisan Bias Modulate Neural Processing of Political Information? An Analysis of the Neural Correlates of Corruption and Positive Messages. Political Psychology, 41(1), 145-164. https://doi.org/10.1111/pops.12581

Cascio, C. N., Scholz, C., \& Falk, E. B. (2015). Social influence and the brain: Persuasion, susceptibility to influence and retransmission. Current Opinion in Behavioral Sciences, 3, 51-57. https://doi.org/10.1016/j.cobeha.2015.01.007

Falk, E. B., Way, B. M., \& Jasinska, A. J. (2012). An imaging genetics approach to understanding social influence. Frontiers in Human Neuroscience, 6(JUNE 2012), 1-13. https://doi.org/10.3389/fnhum.2012.00168

Li, L., Li, K. K., \& Li, J. (2019). Private but not social information validity modulates social conformity bias. Human Brain Mapping, 40(8), 2464-2474. https://doi.org/10.1002/hbm.24536

Sherman, L. E., Payton, A. A., Hernandez, L. M., Greenfield, P. M., \& Dapretto, M. (2016). The Power of the Like in Adolescence: Effects of Peer Influence on Neural and Behavioral Responses to Social Media. Psychological Science, 27(7), 1027-1035. https://doi.org/10.1177/0956797616645673

Baek, E. C., O’Donnell, M. B., Scholz, C., Pei, R., Garcia, J. O., Vettel, J. M., \& Falk, E. B. (2021). Activity in the brain's valuation and mentalizing networks is associated with propagation of online recommendations. Scientific Reports, 11(1), 1-11. https://doi.org/10.1038/s41598-021-90420-2

Nakao, T., Kanayama, N., Katahira, K., Odani, M., Ito, Y., Hirata, Y., Nasuno, R., Ozaki, H., Hiramoto, R., Miyatani, M., \& Northoff, G. (2016). Post-response $\beta$ y power predicts the degree of choice-based learning in internally guided decision-making. Scientific Reports, 6(August), 1-9. https://doi.org/10.1038/srep32477

Kappes, A., Harvey, A. H., Lohrenz, T., Montague, P. R., \& Sharot, T. (2020). Confirmation bias in the utilization of others' opinion strength. Nature Neuroscience, 23(1), 130-137. https://doi.org/10.1038/s41593-019-0549-2

Izuma, K., \& Adolphs, R. (2013). Social manipulation of preference in the human brain. Neuron, 78(3), 563-573. https://doi.org/10.1016/j.neuron.2013.03.023

Klucharev, V., Munneke, M. A. M., Smidts, A., \& Fernández, G. (2011). Downregulation of the posterior medial frontal cortex prevents social conformity. Journal of Neuroscience, 31(33), 11934-11940. https://doi.org/10.1523/JNEUROSCI.1869-11.2011

Garcia, J. O., Ashourvan, A., Muldoon, S., Vettel, J. M., \& Bassett, D. S. (2018). Applications of Community Detection Techniques to Brain Graphs: Algorithmic 
Considerations and Implications for Neural Function. Proceedings of the IEEE, 106(5), 846-867. https://doi.org/10.1109/JPROC.2017.2786710.

Bassett, D. S., Wymbs, N. F., Porter, M. A., Mucha, P. J., Carlson, J. M., \& Grafton, S. T. (2011). Dynamic reconfiguration of human brain networks during learning. Proceedings of the National Academy of Sciences of the United States of America, 108(18), 7641-7646. https://doi.org/10.1073/pnas.1018985108.

Bassett, D. S., Wymbs, N. F., Rombach, M. P., Porter, M. A., Mucha, P. J., \& Grafton, S. T. (2013). Task-Based Core-Periphery Organization of Human Brain Dynamics. PLoS Computational Biology, 9(9), 1-16. https://doi.org/10.1371/journal.pcbi.1003171

Gerraty, R. T., Davidow, J. Y., Foerde, K., Galvan, A., Bassett, D. S., \& Shohamy, D. (2018). Dynamic flexibility in striatal-cortical circuits supports reinforcement learning. Journal of $\quad 38(10)$, https://doi.org/10.1523/JNEUROSCI.2084-17.2018

Reddy, P. G., Mattar, M. G., Murphy, A. C., Wymbs, N. F., Grafton, S. T., Satterthwaite, T. D., \& Bassett, D. S. (2018). Brain state flexibility accompanies motor-skill acquisition. Neurolmage, 171(December 2017), 135-147. https://doi.org/10.1016/j.neuroimage.2017.12.093

Alavash, M., Hilgetag, C. C., Thiel, C. M., \& Gießing, C. (2015). Persistency and flexibility of complex brain networks underlie dual-task interference. Human Brain Mapping, 36(9), 3542-3562. https://doi.org/10.1002/hbm.22861

Shafiei, S. B., Elsayed, A. S., Hussein, A. A., Iqbal, U., \& Guru, K. A. (2020). Evaluating the Mental Workload During Robot-Assisted Surgery Utilizing Network Flexibility of Human Brain. IEEE Access, 8, 204012-204019. https://doi.org/10.1109/access.2020.3036751

Thomas Yeo, B. T., Krienen, F. M., Eickhoff, S. B., Yaakub, S. N., Fox, P. T., Buckner, R. L., Asplund, C. L., \& Chee, M. W. L. (2015). Functional specialization and flexibility in human association cortex. Cerebral Cortex, 25(10), 3654-3672. https://doi.org/10.1093/cercor/bhu217

Telesford, Q. K., Lynall, M. E., Vettel, J., Miller, M. B., Grafton, S. T., \& Bassett, D. S. (2016). Detection of functional brain network reconfiguration during task-driven cognitive states. Neurolmage, 142, 198-210. https://doi.org/10.1016/j.neuroimage.2016.05.078

Chai, L. R., Mattar, M. G., Blank, I. A., Fedorenko, E., \& Bassett, D. S. (2016). Functional Network Dynamics of the Language System. Cerebral Cortex, 26(11), 4148-4159. https://doi.org/10.1093/cercor/bhw238

Han, S., Cui, Q., Wang, X., Li, L., Li, D., He, Z., Guo, X., Fan, Y. S., Guo, J., Sheng, W., Lu, F., \& Chen, H. (2020). Resting state functional network switching rate is differently altered in bipolar disorder and major depressive disorder. Human Brain Mapping, 41(12), 3295-3304. https://doi.org/10.1002/hbm.25017 
Lydon-Staley, D. M., Kuehner, C., Zamoscik, V., Huffziger, S., Kirsch, P., \& Bassett, D. S. (2019). Repetitive negative thinking in daily life and functional connectivity among default mode, fronto-parietal, and salience networks. Translational Psychiatry, 9(1). https://doi.org/10.1038/s41398-019-0560-0

Paban, V., Modolo, J., Mheich, A., \& Hassan, M. (2019). Psychological resilience correlates with EEG source-space brain network flexibility. Network Neuroscience, 3(2), 539-550. https://doi.org/10.1162/netn_a_00079

Betzel, R. F., \& Bassett, D. S. (2017). Multi-scale brain networks. Neurolmage, 160(November 2016), 73-83. https://doi.org/10.1016/j.neuroimage.2016.11.006

Braun, U., Schäfer, A., Walter, H., Erk, S., Romanczuk-Seiferth, N., Haddad, L., Schweiger, J. I., Grimm, O., Heinz, A., Tost, H., Meyer-Lindenberg, A., \& Bassett, D. S. (2015). Dynamic reconfiguration of frontal brain networks during executive cognition in humans. Proceedings of the National Academy of Sciences of the United States of America, 112(37), 11678-11683. https://doi.org/10.1073/pnas.1422487112

Lauharatanahirun, N., Bansal, K., Thurman, S. M., Vettel, J. M., Giesbrecht, B., Grafton, S., Elliott, J. C., Flynn-Evans, E., Falk, E., \& Garcia, J. O. (2020). Flexibility of brain regions during working memory curtails cognitive consequences to lack of sleep. i. http://arxiv.org/abs/2009.07233

Barbey, A. K. (2018). Network Neuroscience Theory of Human Intelligence. Trends in Cognitive Sciences, 22(1), 8-20. https://doi.org/10.1016/j.tics.2017.10.001

Imperatori, L. S., Betta, M., Cecchetti, L., Canales-Johnson, A., Ricciardi, E., Siclari, F., Pietrini, P., Chennu, S., \& Bernardi, G. (2019). EEG functional connectivity metrics wPLI and wSMI account for distinct types of brain functional interactions. Scientific Reports, 9(1), 1-15. https://doi.org/10.1038/s41598-019-45289-7.

Vinck, M., Oostenveld, R., Van Wingerden, M., Battaglia, F., \& Pennartz, C. M. A. (2011). An improved index of phase-synchronization for electrophysiological data in the presence of volume-conduction, noise and sample-size bias. Neurolmage, 55(4), 1548-1565. https://doi.org/10.1016/j.neuroimage.2011.01.055.

Bassett, D. S., \& Sporns, O. (2017). Network neuroscience. Nature Neuroscience, 20(3), 353-364. https://doi.org/10.1038/nn.4502.

Mucha, P. J., Richardson, T., Macon, K., Porter, M. A., \& Onnela, J. P. (2010). Community structure in time-dependent, multiscale, and multiplex networks. Science, 328(5980), 876-878. https://doi.org/10.1126/science.1184819.

Blondel, V. D., Guillaume, J. L., Lambiotte, R., \& Lefebvre, E. (2008). Fast unfolding of communities in large networks. Journal of Statistical Mechanics: Theory and Experiment, 2008(10). https://doi.org/10.1088/1742-5468/2008/10/P10008. 
Good, B. H., De Montjoye, Y. A., \& Clauset, A. (2010). Performance of modularity maximization in practical contexts. Physical Review E - Statistical, Nonlinear, and Soft Matter Physics, 81(4), 1-20. https://doi.org/10.1103/PhysRevE.81.046106.

Richard, C., Karić, M. S., McConnell, M., Poole, J., Rupp, G., Fink, A., Meghdadi, A., \& Berka, C. (2021). Elevated Inter-Brain Coherence Between Subjects With Concordant Stances During Discussion of Social Issues. Frontiers in Human Neuroscience, 15(May), 1-11. https://doi.org/10.3389/fnhum.2021.611886

Hardmeier, M., Hatz, F., Bousleiman, H., Schindler, C., Stam, C. J., \& Fuhr, P. (2014). Reproducibility of functional connectivity and graph measures based on the phase lag index (PLI) and weighted phase lag index (WPLI) derived from high resolution EEG. PLoS ONE, 9(10). https://doi.org/10.1371/journal.pone.0108648

Uddin, L. Q. (2020). Bring the Noise: Reconceptualizing Spontaneous Neural Activity. Trends in Cognitive Sciences, 24(9), 734-746. https://doi.org/10.1016/i.tics.2020.06.003

Doron, K. W., Bassett, D. S., \& Gazzaniga, M. S. (2012). Dynamic network structure of interhemispheric coordination. Proceedings of the National Academy of Sciences of the United States of America, 109(46), 18661-18668. https://doi.org/10.1073/pnas.1216402109

Rilling, J. K., \& Sanfey, A. G. (2011). The neuroscience of social decision-making. $\begin{array}{lllll}\text { Annual review } & \text { of } & \text { 23-48. }\end{array}$ https://doi.org/10.1146/annurev.psych.121208.131647

Wallace, K. J., \& Hofmann, H. A. (2021). Decision-making in a social world: Integrating cognitive ecology and social neuroscience. Current Opinion in Neurobiology, 68, 152-158. https://doi.org/10.1016/j.conb.2021.03.009

Shine, J. M., \& Poldrack, R. A. (2018). Principles of dynamic network reconfiguration across diverse brain states. Neurolmage, 180(March 2017), 396-405. https://doi.org/10.1016/j.neuroimage.2017.08.010

Cooper, N., Garcia, J. O., Tompson, S. H., O’Donnell, M. B., Falk, E. B., \& Vettel, J. M. (2019). Time-evolving dynamics in brain networks forecast responses to health messaging. Network Neuroscience, 3(1), 138-156. https://doi.org/10.1162/netn a 00058

Yin, D., \& Kaiser, M. (2021). Understanding neural flexibility from a multifaceted $\begin{array}{lll}\text { definition. } & \text { Neurolmage, 235(October } & 2020),\end{array}$ https://doi.org/10.1016/j.neuroimage.2021.118027

Mattar, M. G., Betzel, R. F., \& Bassett, D. S. (2016). The flexible brain. Brain, 139(8), 2110-2112. https://doi.org/10.1093/brain/aww151

Hanes, K., Andrewes, D., \& Pantelis, C. (1995). Cognitive flexibility and complex integration in Parkinson's disease, Huntington's disease, and Schizophrenia. Journal 
of the International Neuropsychological Society, 1(6), 545-553. https://doi.org/10.1017/S1355617700000679

Jirsa, V. K., Stacey, W. C., Quilichini, P. P., Ivanov, A. I., \& Bernard, C. (2014). On the nature of seizure dynamics. Brain, 137(8), 2210-2230. https://doi.org/10.1093/brain/awu133

Rungratsameetaweemana, N., Lainscsek, C., Cash, S. S., Garcia, J. O., Sejnowski, T. J., \& Bansal, K. (2021). Brain network dynamics codify heterogeneity in seizure propagation. bioRxiv. https://doi.org/10.1101/2021.06.12.448205

Ruelle, D. (1976). The Lorenz attractor and the problem of turbulence. In Turbulence and Navier Stokes Equations (pp. 146-158). Springer, Berlin, Heidelberg.

Pikovsky, A., Rosenblum, M., Kurths, J., \& Synchronization, A. (2001). A universal concept in nonlinear sciences. Self, 2, 3.

Strogatz, S. H. (2000). From Kuramoto to Crawford: Exploring the onset of synchronization in populations of coupled oscillators. Physica D: Nonlinear Phenomena, 143(1-4), 1-20. https://doi.org/10.1016/S0167-2789(00)00094-4

Holley, R. A., \& Liggett, T. M. (1975). Ergodic theorems for weakly interacting infinite systems and the voter model. The annals of probability, 643-663.

Krapivsky, P. L., \& Redner, S. (2003). Dynamics of Majority Rule in Two-State Interacting Spin Systems. Physical Review Letters, 90(23), 4. https://doi.org/10.1103/PhysRevLett.90.238701

Cocchi, L., Gollo, L. L., Zalesky, A., \& Breakspear, M. (2017). Criticality in the brain: A synthesis of neurobiology, models and cognition. Progress in Neurobiology, 158, 132-152. https://doi.org/10.1016/j.pneurobio.2017.07.002

Mahmoodi, K., West, B. J., \& Grigolini, P. (2017). Self-organizing complex networks: Individual versus global rules. Frontiers in Physiology, 8(JUL), 1-11. https://doi.org/10.3389/fphys.2017.00478

Bansal, K., Garcia, J. O., Lauharatanahirun, N., Muldoon, S. F., Sajda, P., \& Vettel, J. M. (2021). Scale-specific dynamics of high-amplitude bursts in EEG capture behaviorally meaningful variability. Neurolmage, 241(June), 118425. https://doi.org/10.1016/j.neuroimage.2021.118425

Buzsaki, Gyorgy. Rhythms of the Brain. Oxford university press, 2006.

Bragin, A., Jandó, G., Nádasdy, Z., Hetke, J., Wise, K., \& Buzsáki, G. (1995). Gamma (40-100 Hz) oscillation in the hippocampus of the behaving rat. Journal of neuroscience, 15(1), 47-60. https://doi.org/10.1523/JNEUROSCl.15-01-00047.1995

Chrobak, J. J., \& Buzsáki, G. (1998). Gamma oscillations in the entorhinal cortex of the freely behaving rat. Journal of Neuroscience, 18(1), 388-398. https://doi.org/10.1523/jneurosci.18-01-00388.1998 
Leopold, D. A., Murayama, Y., \& Logothetis, N. K. (2003). Very slow activity fluctuations in monkey visual cortex: Implications for functional brain imaging. Cerebral Cortex, 13(4), 422-433. https://doi.org/10.1093/cercor/13.4.422

Schroeder, C. E., \& Lakatos, P. (2009). The Gamma Oscillation: Master or Slave? Brain Topography, 22(1), 24-26. https://doi.org/10.1007/s10548-009-0080-y

Canolty, R. T., Edwards, E., Dalal, S. S., Soltani, M., Nagarajan, S. S., Berger, M. S., Barbaro, N. M., \& Knight, R. T. (2009). NIH Public Access. 313(5793), 1626-1628. https://doi.org/10.1126/science.1128115. High

Buzsáki, G., \& Wang, X. J. (2012). Mechanisms of gamma oscillations. Annual review of neuroscience,

35 ,

203-225.

https://doi.org/10.1146/annurev-neuro-062111-150444

Cavanagh, J. \& Frank, M. J. (2014). Frontal theta as a mechanism for cognitive control. Trends Cogn Sci, 18(8), 414-21. https://doi.org/10.1016/j.tics.2014.04.012

Rungratsameetaweemana, N., Itthipuripat, S., Salazar, A., \& Serences, J. T. (2018). Expectations do not alter early sensory processing during perceptual decision-making. Journal of Neuroscience, 38(24), 5632-5648. https://doi.org/10.1523/JNEUROSCI.3638-17.2018

Khanna, P., \& Carmena, J. M. (2015). Neural oscillations: Beta band activity across motor networks. Current Opinion in Neurobiology, 32, 60-67. https://doi.org/10.1016/j.conb.2014.11.010

Engel, A. K., \& Fries, P. (2010). Beta-band oscillations-signalling the status quo? Current Opinion in Neurobiology, 20(2), 156-165. https://doi.org/10.1016/j.conb.2010.02.015

Haegens, S., Händel, B. F., \& Jensen, O. (2011). Top-down controlled alpha band activity in somatosensory areas determines behavioral performance in a discrimination task. Journal of Neuroscience, 31(14), 5197-5204. https://doi.org/10.1523/JNEUROSCl.5199-10.2011

Nakao, T., Miyagi, M., Hiramoto, R., Wolff, A., Gomez-Pilar, J., Miyatani, M., \& Northoff, G. (2019). From neuronal to psychological noise - Long-range temporal correlations in EEG intrinsic activity reduce noise in internally-guided decision making. Neurolmage, $\quad 201$ (June), 116015. https://doi.org/10.1016/i.neuroimage.2019.116015

Golnar-Nik, P., Farashi, S., \& Safari, M. S. (2019). The application of EEG power for the prediction and interpretation of consumer decision-making: A neuromarketing study. Physiology and Behavior, 207(April), 90-98. https://doi.org/10.1016/j.physbeh.2019.04.025

Sugimura, K., Iwasa, Y., Kobayashi, R., Honda, T., Hashimoto, J., Kashihara, S., Zhu, J., Yamamoto, K., Kawahara, T., Anno, M., Nakagawa, R., Hatano, K., \& 
Nakao, T. (2021). Association between long-range temporal correlations in intrinsic EEG activity and subjective sense of identity. Scientific Reports, 11(1), 422. https://doi.org/10.1038/s41598-020-79444-2

Wehrens, R., Putter, H., \& Buydens, L. M. C. (2000). The bootstrap: A tutorial. Chemometrics and Intelligent Laboratory Systems, 54(1), 35-52. https://doi.org/10.1016/S0169-7439(00)00102-7

\section{Supplemental Material}

\section{EEG sensor position}

The EEG recordings were obtained using the B-Alert $\mathrm{R} X 24$ wireless sensor headset (Advanced Brain Monitoring, Inc., Carlsbad, CA, United States), the system has 20 channels and the montage layout is presented in (Figure S1). The reference sensors were located behind each ear on the mastoid bone. The sample rate was $256 \mathrm{~Hz}$ with a high band pass at $0.1 \mathrm{~Hz}$ and a low band bass at $100 \mathrm{~Hz}$. 


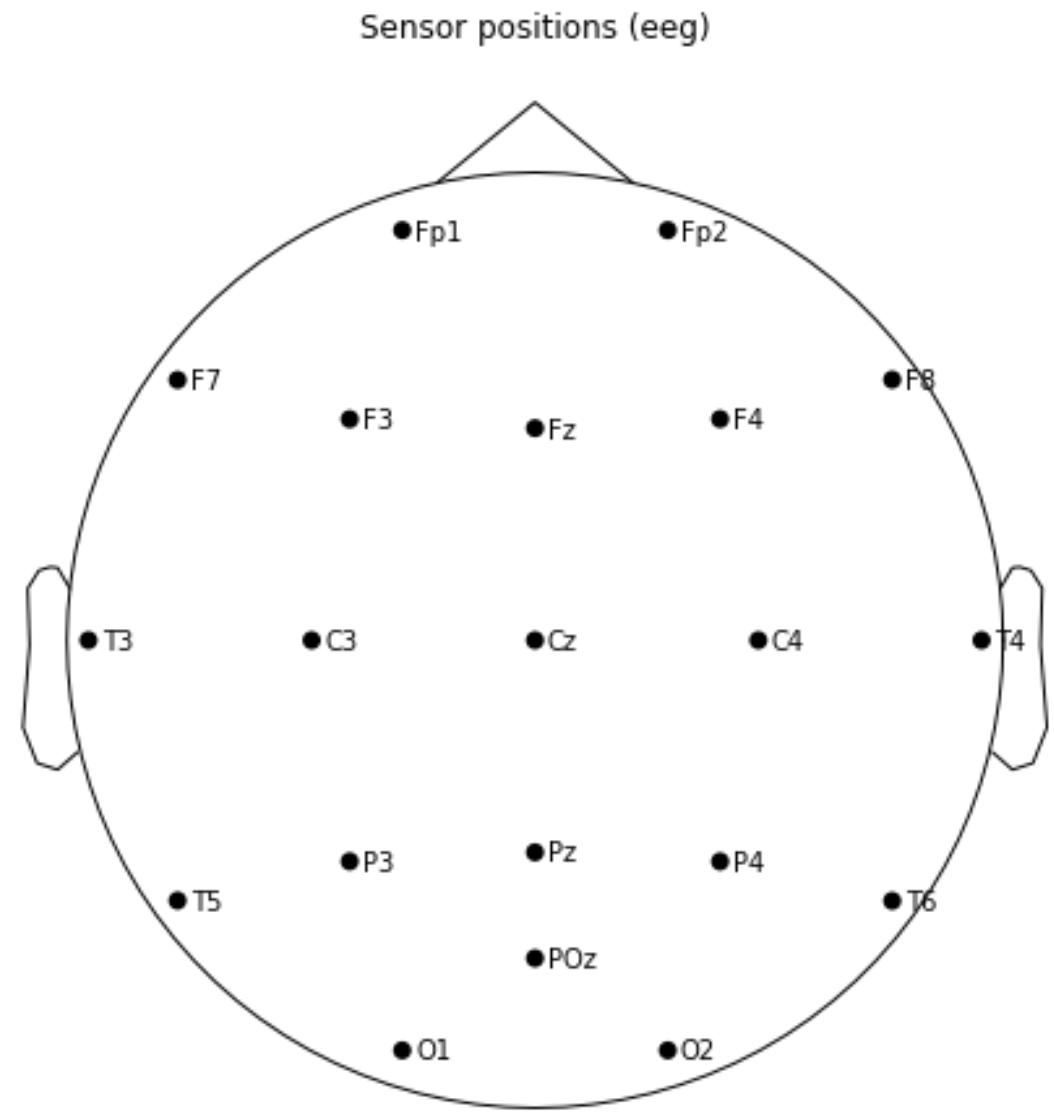

Figure S1: EEG sensor position. The topo plot shows the sensor montage. The EEG recording system model used was B-Alert R X24 wireless sensor headset (Advanced Brain Monitoring, Inc., Carlsbad, CA, United States) with 20 channels.

\section{Node allegiance}

To further understand the differences between rigid and malleable opinionators in terms of network reconfigurations, we explored how the network nodes, particularly the nodes that showed significant difference in flexibility between two groups, (significant nodes, Figure 2) changed their functional allegiances. Allegiance is defined as the fraction of the total time two nodes are in the same community. First, we calculated the allegiance metric between all the node pairs and then compared them between two groups. None of the node pairs (including or excluding the significant nodes) showed a significant difference between rigid and malleable opinionators. Whereas, on an average, some of the node-pairs showed higher allegiances for rigid opinionators while some showed higher allegiances for the malleable opinionators. In Figure S2, we show average allegiance differences between two groups. Yellow entries in the matrices represent higher allegiances for rigid opinionators. In topographical plots we show these differences only for the significant nodes. 

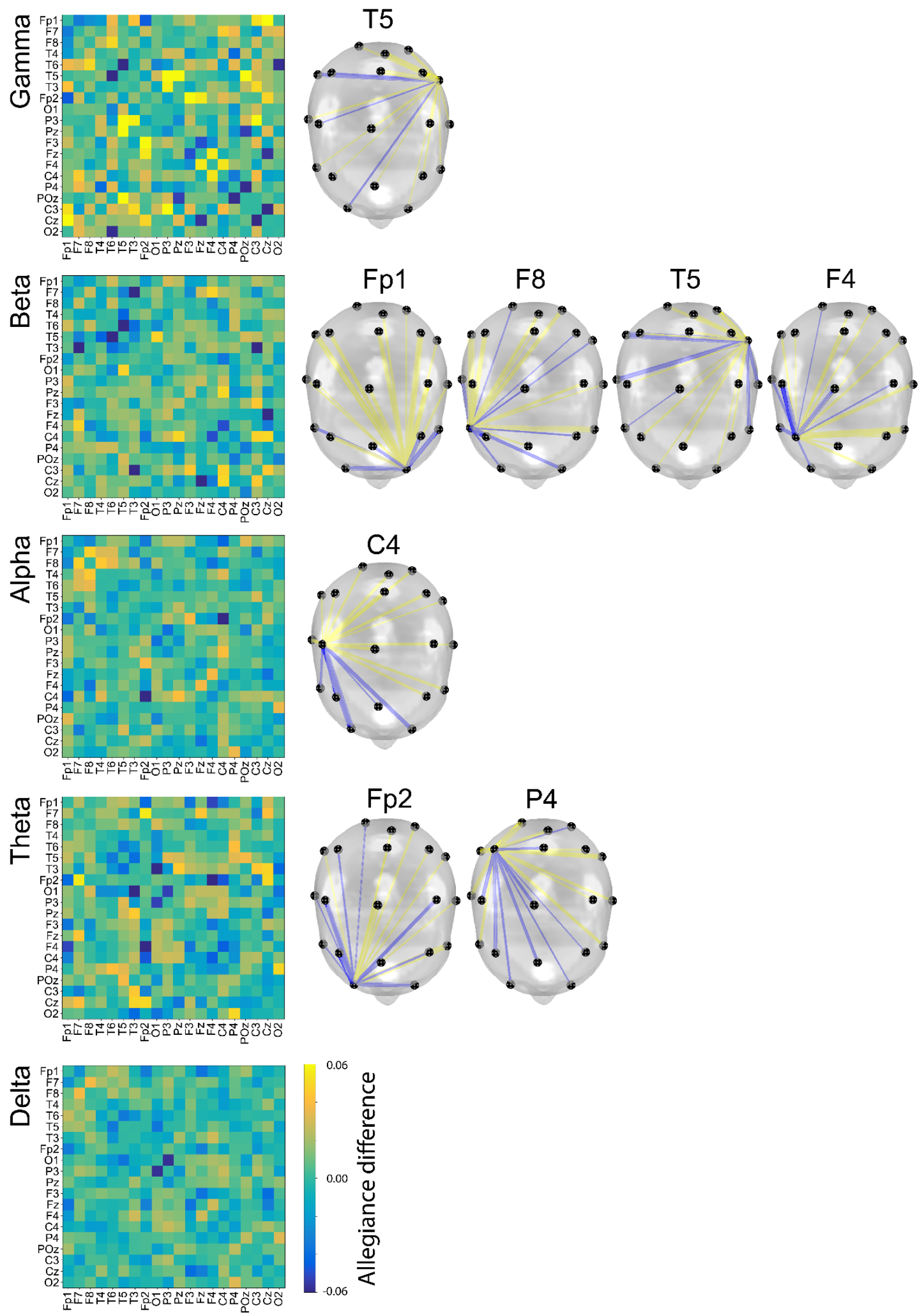

Figure S2: Node allegiance differences. The matrices show average allegiance differences between the two groups (rigid and malleable opinionators). The topo plots show the mean allegiance differences for sensors which showed significant 
flexibility changes between the two groups (as discussed in Figure 2). The links in yellow (blue) indicate a higher (lower) allegiance value for the rigid (malleable) group.

\section{Consensus community structure}

We calculated the consensus community structure for the time layers of each subject and estimated the mean and standard deviation for degrees both within and across communities. This procedure generated two communities, one in the frontal region with 11 sensors (Fp1, F7, F8, T3, Fp2, F3, Fz, F4, C4, C3, Cz) and the other on the rear region with 9 sensors $(\mathrm{T} 4, \mathrm{~T} 6, \mathrm{~T} 5, \mathrm{O} 1, \mathrm{P} 3, \mathrm{Pz}, \mathrm{P} 4, \mathrm{POz}, \mathrm{O} 2)$. The topo plot is presented in (Figure S3)

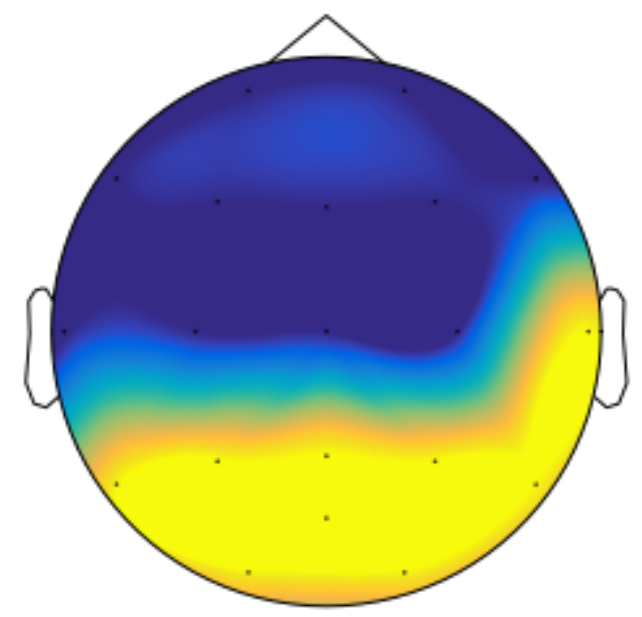

Figure S3: Consensus community structures. The topo plot shows the regions of the two communities obtained through consensus on the entire cohort of subjects. First was calculated the consensus community of the time layers for each subject, the resulting community structure was used to obtain the consensus community structure for the entire cohort of subjects. 

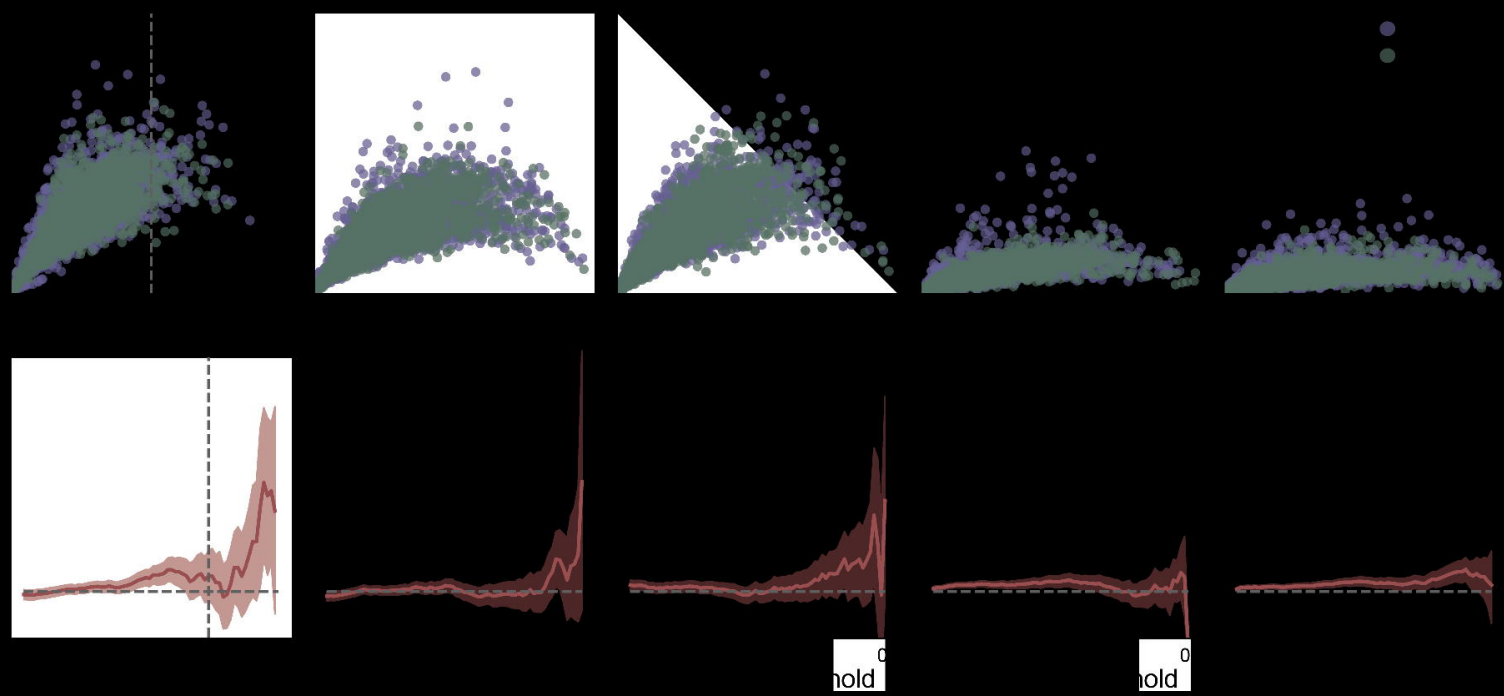

I

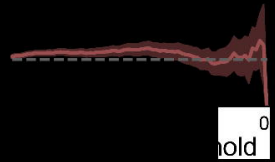

I

口 


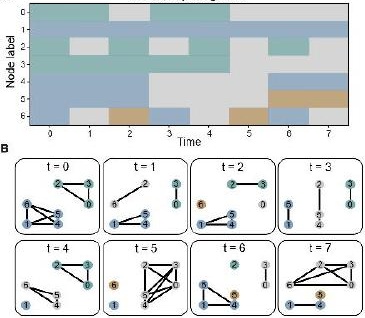




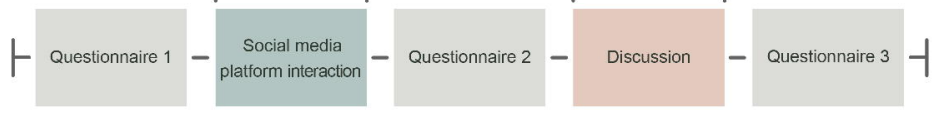

\section{Social media platform opinion change}

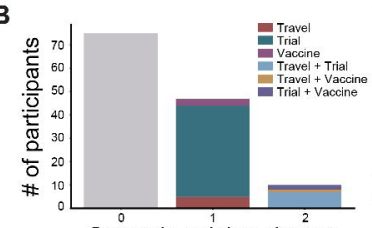

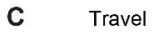

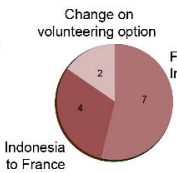

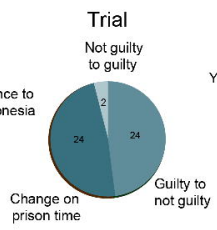

\section{Trial}

Scenario opinion change

\section{Vaccine}

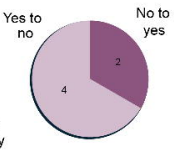

\section{Discussion opinion change}

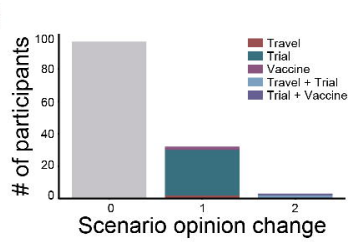

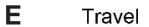

Change on

volunteering option

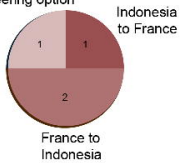

Trial

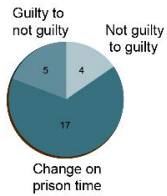

Vaccine

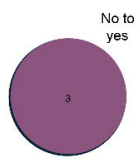

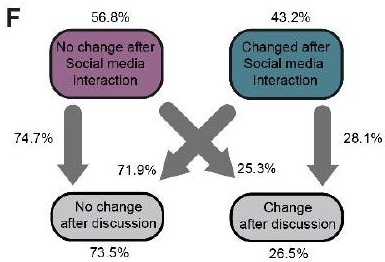




\section{Delta}

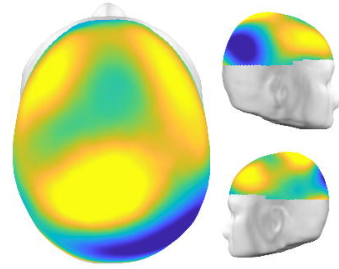

B
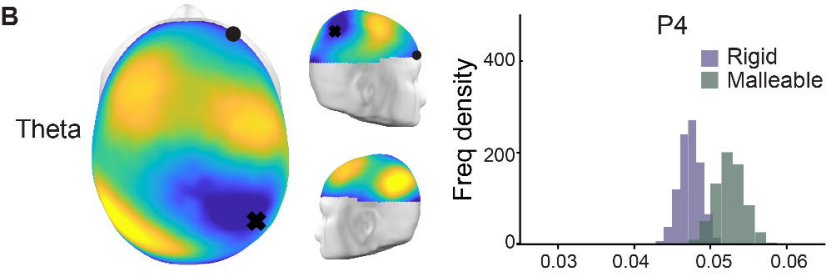

Alpha

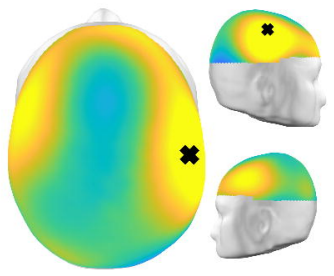

D

C

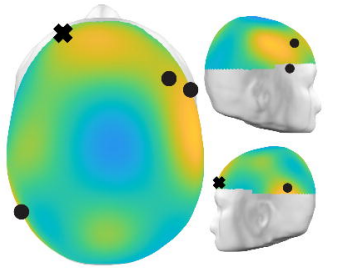

E

Gamma

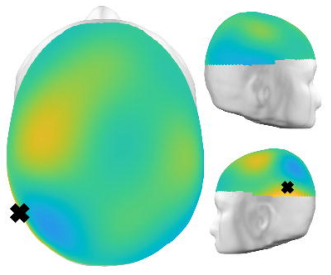

$-5 \times 10^{-3}$

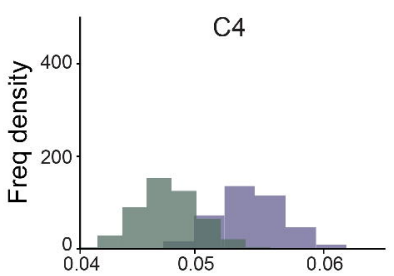

Fp1

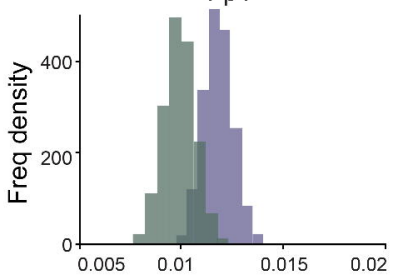

T5

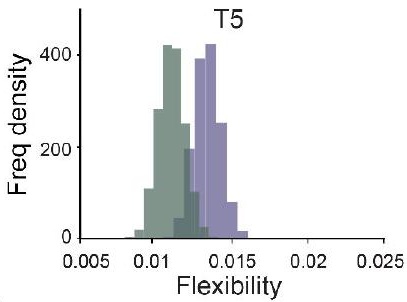

Flexibility difference 


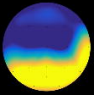



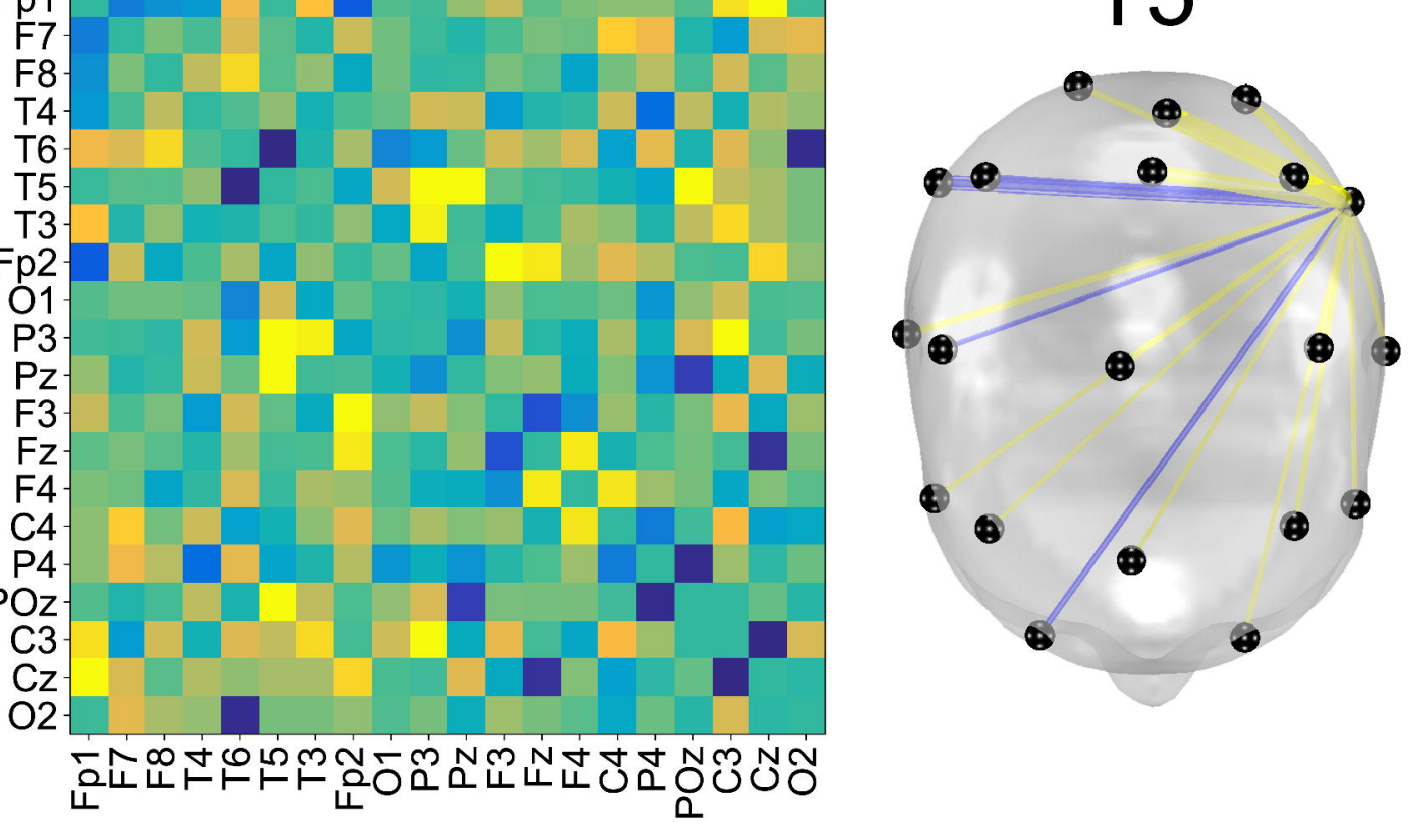

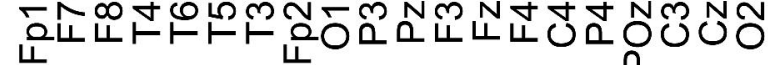

$\underset{\varpi}{\mathbb{D}}$

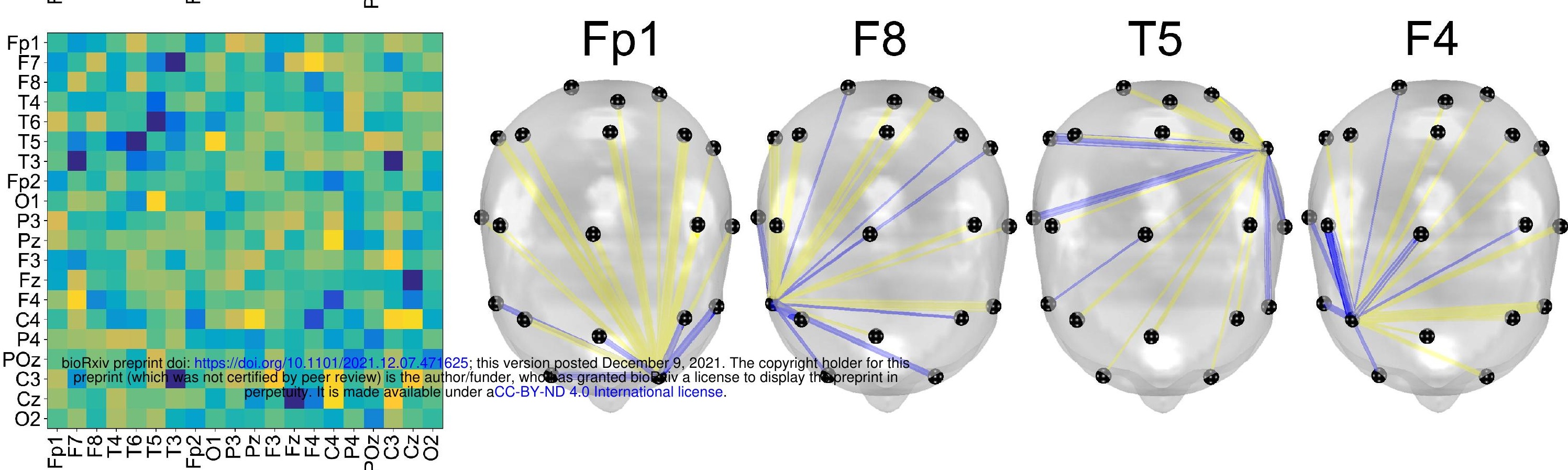

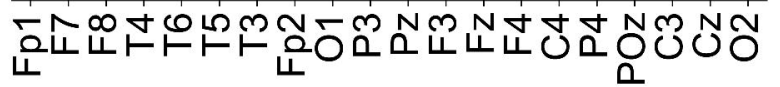

$\frac{\frac{\sigma}{c}}{\frac{1}{1}}$
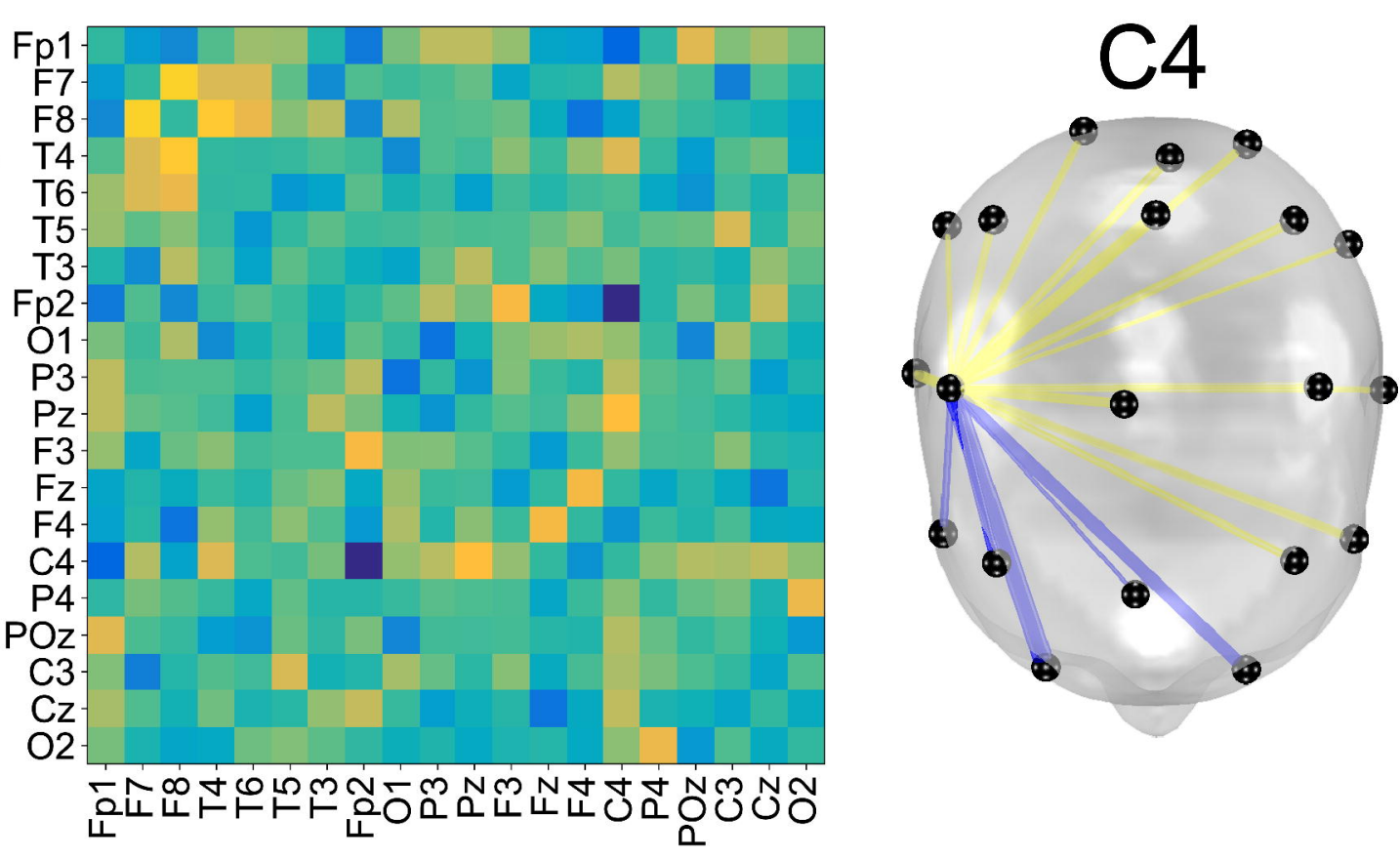


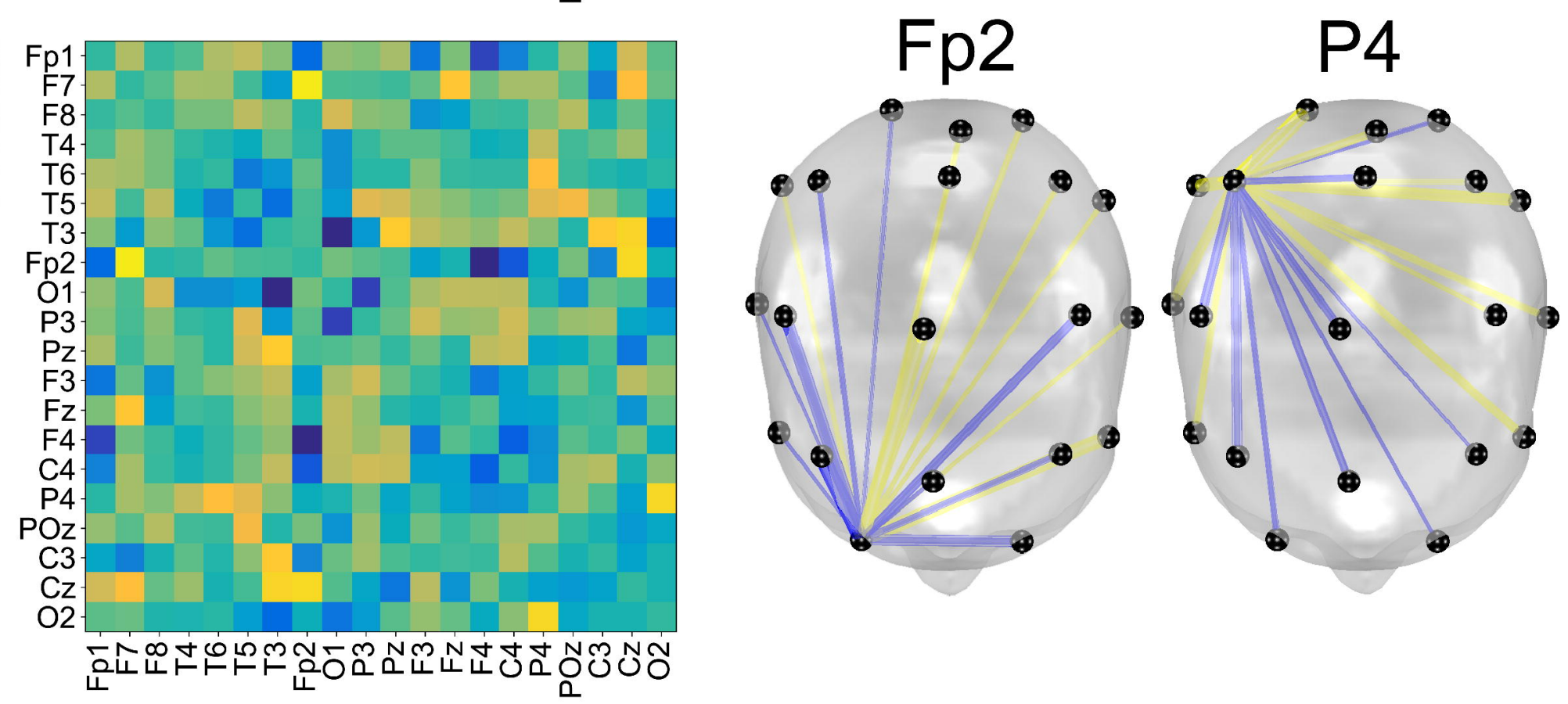

$\frac{\text { (] }}{\frac{1}{0}}$

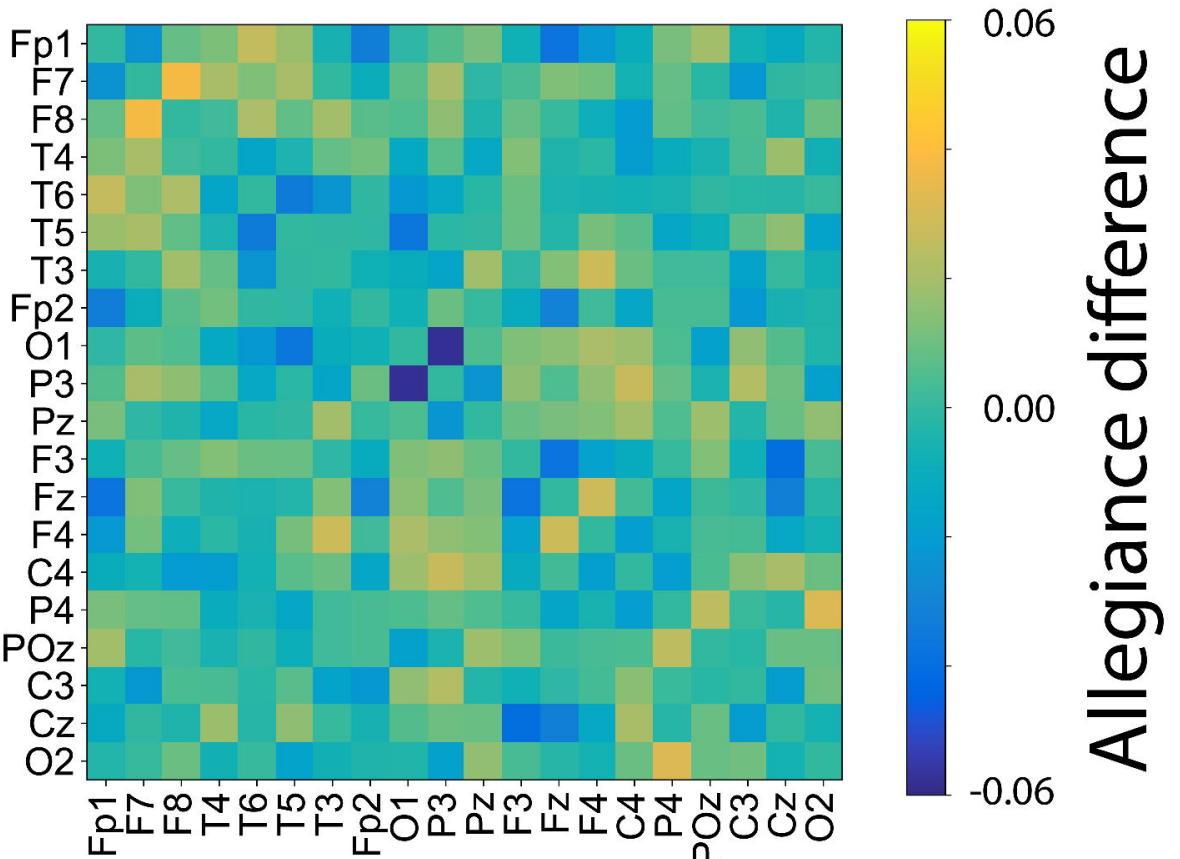


Sersor positions (eeg)

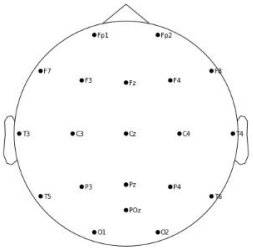

